\title{
Thermodynamics of spin chains of Haldane-Shastry type and one-dimensional vertex models
}

\author{
Alberto Enciso $^{\mathrm{a}}$, Federico Finkel ${ }^{\mathrm{b}}$, Artemio González-López ${ }^{\mathrm{b}, *}$ \\ ${ }^{a}$ Instituto de Ciencias Matemáticas, Consejo Superior de Investigaciones Científicas, 28049 Madrid, Spain \\ ${ }^{b}$ Departamento de Física Teórica II, Universidad Complutense de Madrid, 28040 Madrid, Spain
}

\begin{abstract}
We study the thermodynamic properties of spin chains of Haldane-Shastry type associated with the $A_{N-1}$ root system in the presence of a uniform external magnetic field. To this end, we exactly compute the partition function of these models for an arbitrary finite number of spins. We then show that these chains are equivalent to a suitable inhomogeneous classical Ising model in a spatially dependent magnetic field, generalizing the results of Basu-Mallick et al. for the zero magnetic field case. Using the standard transfer matrix approach, we are able to compute in closed form the free energy per site in the thermodynamic limit. We perform a detailed analysis of the chains' thermodynamics in a unified way, with special emphasis on the zero field and zero temperature limits. Finally, we provide a novel interpretation of the thermodynamic quantities of spin chains of Haldane-Shastry type as weighted averages of the analogous quantities over an ensemble of classical Ising models.
\end{abstract}

Keywords: Spin chains of Haldane-Shastry type, vertex models, transfer matrix method, thermodynamic limit

\section{Introduction}

In this paper we study a class of $\operatorname{su}(m)$ spin chains whose Hamiltonian can be collectively written as

$$
\mathcal{H}=\sum_{1 \leqslant i<j \leqslant N} J_{i j}\left(1-S_{i j}\right)-\sum_{\alpha=1}^{m-1} B_{\alpha} S^{\alpha},
$$

where the $B_{\alpha}$ 's are real constants and the interactions $J_{i j}$ are chosen as described below (see Eqs. (1.7)). In the previous formula, the operators $S_{i j}$ act on a state

$$
\left|s_{1}, \ldots, s_{N}\right\rangle, \quad s_{i} \in\{1, \ldots, m\},
$$

of the canonical spin basis by permuting the $i$-th and $j$-th spins:

$$
S_{i j}\left|s_{1}, \ldots, s_{i}, \ldots, s_{j}, \ldots, s_{N}\right\rangle=\left|s_{1}, \ldots, s_{j}, \ldots, s_{i}, \ldots, s_{N}\right\rangle .
$$

${ }^{*}$ Corresponding author 
The permutation operators $S_{i j}$ can be expressed in terms of the (Hermitian) su( $\left.m\right)$ spin operators $T_{k}^{\alpha}$ with the normalization $\operatorname{tr}\left(T_{k}^{\alpha} T_{k}^{\beta}\right)=2 \delta^{\alpha \beta}$ (where the subindex $k$ labels the chain sites) as [1]

$$
S_{i j}=\frac{1}{m}+\frac{1}{2} \sum_{\alpha=1}^{m^{2}-1} T_{i}^{\alpha} T_{j}^{\alpha} .
$$

Let $S_{k}^{\alpha}$ denote the operator whose action on the canonical spin basis is given by

$$
S_{k}^{\alpha}\left|s_{1}, \ldots, s_{N}\right\rangle=\left(\delta_{s_{k}}^{\alpha}-\delta_{s_{k}}^{m}\right)\left|s_{1}, \ldots, s_{N}\right\rangle, \quad \alpha=1, \ldots, m-1,
$$

so that the operators $i S_{k}^{\alpha}$ span the standard Cartan subalgebra of $\operatorname{su}(m)$. The operators $S^{\alpha}$ are then defined by

$$
S^{\alpha}=\sum_{i=1}^{N} S_{i}^{\alpha}, \quad \alpha=1, \ldots, m-1 .
$$

Thus the second sum in Eq. (1.1) can be interpreted as arising from the interaction with a uniform external su $(m)$ "magnetic" field 1 of strengths $\left(B_{1}, \ldots, B_{m-1}\right)$. Note that in the case $m=2$ (i.e., for spin 1/2) we can take $\left\{T_{k}^{1}, T_{k}^{2}, T_{k}^{3}\right\}=\left\{\sigma_{k}^{x}, \sigma_{k}^{y}, \sigma_{k}^{z}\right\}$ and $S_{k}^{1}=\sigma_{k}^{z}$, where $\sigma_{k}^{v}$ is a Pauli matrix acting on the $k$-th spin's Hilbert space. Hence Eq. (1.2) adopts the more familiar form

$$
S_{i j}=\frac{1}{2}\left(1+\sigma_{i} \cdot \sigma_{j}\right)
$$

and the Hamiltonian 1.1 reduces to

$$
\mathcal{H}=\sum_{1 \leqslant i<j \leqslant N} \frac{J_{i j}}{2}\left(1-\boldsymbol{\sigma}_{i} \cdot \boldsymbol{\sigma}_{j}\right)-B \sum_{i=1}^{N} \sigma_{i}^{z},
$$

with $B \equiv B_{1}$. In particular, the last term represents the interaction with a uniform magnetic field parallel to the $z$ axis with strength (proportional to) $B$.

The three models we shall study are defined by the following choice of the interaction strengths $J_{i j}$ :

- The Haldane-Shastry (HS) chain [2, 3]:

$$
J_{i j}=\frac{J}{2 \sin ^{2}\left(\xi_{i}-\xi_{j}\right)}, \quad \xi_{k}=\frac{k \pi}{N} .
$$

- The Polychronakos-Frahm (PF) chain [4, 5]:

$$
J_{i j}=\frac{J}{\left(\xi_{i}-\xi_{j}\right)^{2}},
$$

${ }^{1}$ The most general su $(m)$ magnetic field term is of the form $T=\sum_{i=1}^{N} T_{i}$, where

$$
T_{i} \equiv \sum_{\alpha=1}^{m^{2}-1} b_{\alpha} T_{i}^{\alpha}
$$

is a traceless Hermitian matrix acting on the internal space of the $i$-th spin. By performing a rotation in this internal space we can diagonalize the matrix $T_{i}$, effectively replacing it by a traceless diagonal matrix. The latter matrix can in turn be expressed in the form $\sum_{\alpha=1}^{m-1} B_{\alpha} S_{i}^{\alpha}$, which yields the last term in Eq. 1.1. 
where $\xi_{k}$ is the $k$-th root of the Hermite polynomial of degree $N$.

- The Frahm-Inozemtsev (FI) chain [6]:

$$
J_{i j}=\frac{J}{2 \sinh ^{2}\left(\xi_{i}-\xi_{j}\right)},
$$

where $\mathrm{e}^{2 \xi_{k}}$ is the $k$-th root of the generalized Laguerre polynomial $L_{N}^{\alpha-1}$ with $\alpha>0$. In all three cases, $J$ is a real constant whose sign determines the model's ferromagnetic $(J>0)$ or antiferromagnetic $(J<0)$ character. Note that, while the sites of the HS chain are equispaced this is not the case for the PF or FI chains.

We shall denote by

$$
\mathcal{H}_{0}=\sum_{1 \leqslant i<j \leqslant N} J_{i j}\left(1-S_{i j}\right)
$$

the Hamiltonian of the chains (1.1) in the absence of a magnetic field. Following standard terminology, we shall collectively refer to the chains (1.7)-(1.8) as spin chains of Haldane-Shastry type. They are all associated with the root system $A_{N-1}$, in the sense that the interactions $J_{i j}$ depend only on the differences of the site coordinates $\xi_{k}$. Although several generalizations of these chains to the $B C_{N}$ and $D_{N}$ root systems have been considered in the literature [7.-11], in this paper we shall restrict ourselves to the above $A_{N-1}$-type models.

Spin chains of HS type are the simplest models in condensed matter physics exhibiting fractional statistics [12]. Historically, the HS chain (1.7a)-(1.8) was introduced as a simplified version of the one-dimensional Hubbard model with long-range hopping, from which it can be obtained in the half-filling regime when the on-site interaction tends to infinity [13]. Soon after its introduction, it was shown that this chain is completely integrable, in the sense that one can explicitly construct $N-1$ mutually commuting integrals of motion [14, 15]. As first observed by Polychronakos [4], these integrals of motion can be obtained from those of the dynamical spin Sutherland model [16] by means of the so-called "freezing trick". In fact, the freezing trick can also be applied to derive the PF and FI chains from the Calogero [17] and Inozemtsev [18] spin dynamical models. In particular, these two chains are also completely integrable. Apart from their integrable character, spin chains of HS type appear in many areas of current interest in both physics and mathematics, such as quantum chaos [19, 20], supersymmetry [21, 22], conformal field theory [23-25], the AdS-CFT correspondence [26, 27], one-dimensional anyons [28] and Yangian quantum groups [15, 29,-31].

The partition functions of all three chains of HS type in the absence of a magnetic field, which have been computed in closed form using again the freezing trick [32 34], can be expressed in a unified way by the formula

$$
\mathcal{Z}_{0}(q)=\sum_{\mathbf{k} \in \mathcal{P}_{N}} \prod_{i=1}^{r}\left(\begin{array}{c}
m+k_{i}-1 \\
k_{i}
\end{array}\right) \cdot q^{\sum_{i=1}^{r-1} J \mathcal{E}\left(K_{i}\right)} \prod_{i=1}^{N-r}\left(1-q^{J \mathcal{E}\left(K_{i}^{\prime}\right)}\right)
$$

\footnotetext{
${ }^{2}$ In fact, if $2 \xi_{k}$ is interpreted as an angular coordinate, then the HS chain describes an array of spins equispaced on the unit circle, with long-range pairwise interactions inversely proportional to the square of the chord distance between the spins.
} 
Here $q \equiv \mathrm{e}^{-1 /\left(k_{\mathrm{B}} T\right)}, \mathbf{k} \equiv\left(k_{1}, \ldots, k_{r}\right)$ is an element of the set $\mathcal{P}_{N}$ of partitions of $N$ with order taken into account, and the numbers $K_{i}, K_{j}^{\prime} \in\{1, \ldots, N-1\}$ in Eq. [1.9] are positive integers defined by

$$
K_{i}=\sum_{j=1}^{i} k_{j}, \quad\left\{K_{1}^{\prime}, \ldots, K_{N-r}^{\prime}\right\}=\{1, \ldots, N-1\} \backslash\left\{K_{1}, \ldots, K_{r-1}\right\} .
$$

Remarkably, the partition function $\mathcal{Z}_{0}$ of the chains $(1.7)-(1.8)$ depends on the chain under consideration only through its dispersion relation

$$
\mathcal{E}(i)= \begin{cases}i(N-i), & \text { for the HS chain } \\ i, & \text { for the PF chain } \\ i(\alpha+i-1), & \text { for the FI chain. }\end{cases}
$$

Using the previous expression for the partition function, Basu-Mallick et al. [35] showed that the spectrum of the spin chains of HS type is given by

$$
E_{0}(\mathbf{n})=J \sum_{i=1}^{N-1} \delta\left(n_{i}, n_{i+1}\right) \mathcal{E}(i), \quad \mathbf{n} \equiv\left(n_{1}, \ldots, n_{N}\right),
$$

where

$$
\delta(j, k)= \begin{cases}1, & j>k \\ 0, & j \leqslant k\end{cases}
$$

and the quantum numbers $n_{i}$ independently take the values $1, \ldots, m$. The vectors $\delta(\mathbf{n}) \in\{0,1\}^{N-1}$ with components $\delta_{i}(\mathbf{n})=\delta\left(n_{i}, n_{i+1}\right)$ are in fact the celebrated motifs introduced by Haldane et al. [36]. As pointed out in Ref. [35], Eq. [1.12] defines a classical inhomogeneous onedimensional vertex model with bonds $n_{1}, \ldots, n_{N}$ each taking $m$ possible values, where the contribution of the $k$-th vertex is given by $J \delta\left(n_{k-1}, n_{k}\right) \mathcal{E}(k-1)$. We shall show that this connection between spin chains of HS type and vertex models still holds in the presence of a nonzero $\operatorname{su}(m)$ magnetic field. This is indeed the key result on which we shall base our unified approach to the analysis of the thermodynamics of all three chains of HS type (1.1).

The study of the thermodynamic properties of spin chains of HS type was initiated by Haldane himself, who used the spinon description of the spectrum to derive an indirect expression for the entropy of the spin 1/2 HS chain [23]. An explicit formula for the free energy of the PF chain in the absence of a magnetic field appeared shortly afterwards without proof in Ref. [5]. In a subsequent publication [6], Frahm and Inozemtsev obtained an analogous expression for the FI chain using the transfer matrix method, and also computed its magnetization for arbitrary magnetic field.

In this paper we have several objectives that we shall now summarize. In the first place, we shall compute in closed form the partition function of the HS-type chains (1.1) in the presence of an arbitrary $\operatorname{su}(m)$ magnetic field $\left(B_{1}, \ldots, B_{m-1}\right)$. Secondly, we shall use the expression for the partition function to establish the equivalence of the latter chains to a suitable classical inhomogeneous vertex model. We shall then take advantage of this connection to determine the equilibrium thermodynamics of the spin $1 / 2$ chains of HS type in a unified and systematic way.

${ }^{3}$ By Eq. 1.12, the first and last vertices do not contribute to the energy. 
Finally, we shall use the previous results to uncover a novel connection between spin chains of HS type and the classical Ising model.

The paper is organized as follows. In Section 2 we compute the chains' partition functions in closed form by means of the freezing trick. Following the approach of Ref. [35], in Section 3 we construct a generating function for the partition function in terms of complete homogeneous symmetric polynomials. We then define a similar function for the corresponding vertex models, and prove that both generating functions coincide for arbitrary values of their arguments. This shows that the energies of each of the chains (1.1) are identical to those of its associated vertex model. Exploiting this connection, in Section 4 we use the transfer matrix method to find explicit expressions for the thermodynamic functions of the chains (1.1) with spin $1 / 2$. In the case of the $\mathrm{PF}$ chain, we derive an exact formula for the free energy in terms of the dilogarithm function in an arbitrary magnetic field, and show that its magnetization can be expressed in terms of elementary functions. Section 5 is devoted to a detailed study of the zero magnetic field case. In particular, we show that the susceptibility can be expressed for all three chains of HS type in terms of the error function, discuss the connection of these chains with two-level systems, and derive lowtemperature asymptotic expansions the chains' thermodynamic functions. In Section 6 we study the zero temperature limit in the presence of an arbitrary external magnetic field in both the ferromagnetic and antiferromagnetic regimes. We explicitly show that in both regimes there is a phase transition, as was to be expected on general grounds [5]. In Section 7] we present a novel interpretation of the thermodynamic quantities of spin chains of HS type as weighted averages of the analogous quantities over an ensemble of classical Ising chains. The paper ends with a short section where we compare our results with previous related work, and comment on possible extensions thereof.

\section{Partition functions}

In this section we shall derive a closed form expression for the partition functions of the HS chains (1.1) in the presence of a constant $\operatorname{su}(m)$ magnetic field. For definiteness, we shall deal with the PF chain, whose interactions are defined by Eq. (1.7b). As we mentioned above, this chain is obtained by applying the freezing trick to the $\operatorname{su}(m)$ spin Calogero model in a constant magnetic field, whose Hamiltonian we shall take as

$$
H=H_{0}-\frac{2 a}{J} \sum_{\alpha=1}^{m-1} B_{\alpha} S^{\alpha},
$$

where $a>1 / 2$ and

$$
H_{0}=-\sum_{i=1}^{N} \partial_{x_{i}}^{2}+a^{2} \sum_{i=1}^{N} x_{i}^{2}+\sum_{1 \leqslant i \neq j \leqslant N} \frac{a\left(a-S_{i j}\right)}{\left(x_{i}-x_{j}\right)^{2}} .
$$

More precisely, let

$$
H^{\mathrm{sc}}=-\sum_{i=1}^{N} \partial_{x_{i}}^{2}+a^{2} \sum_{i=1}^{N} x_{i}^{2}+\sum_{1 \leqslant i \neq j \leqslant N} \frac{a(a-1)}{\left(x_{i}-x_{j}\right)^{2}}
$$

denote the Hamiltonian of the scalar Calogero model [37], and define

$$
\tilde{\mathcal{H}}(\mathbf{x})=\sum_{1 \leqslant i<j \leqslant N} \frac{1-S_{i j}}{\left(x_{i}-x_{j}\right)^{2}}-\frac{1}{J} \sum_{\alpha=1}^{m-1} B_{\alpha} S^{\alpha},
$$


so that

$$
H=H^{\mathrm{sc}}+2 a \tilde{\mathcal{H}}(\mathbf{x}), \quad \mathcal{H}=J \tilde{\mathcal{H}}(\xi) .
$$

It is well known [38] that the chain sites $\xi$ of the PF chain are the coordinates of the unique minimum of the scalar potential

$$
U(\mathbf{x})=\sum_{i=1}^{N} x_{i}^{2}+\sum_{1 \leqslant i \neq j \leqslant N} \frac{1}{\left(x_{i}-x_{j}\right)^{2}}
$$

in the principal Weyl chamber of $A_{N-1}$ type $x_{1}<x_{2}<\cdots<x_{N}$, where the motion of the particles of the Calogero model can be restricted due to the singularities of its Hamiltonian. From this fact and Eq. 2.1) it follows that in the large $a$ limit the eigenfunctions of the spin Calogero model become sharply peaked around the sites of the PF chain, so that the su(m) degrees of freedom effectively decouple from the dynamical ones. By Eq. [2.5), in this limit the energies of the model (2.1) are approximately given by the formula

$$
E_{i j} \simeq E_{i}^{\mathrm{sc}}+\frac{2 a}{J} e_{j},
$$

where $E_{i}$ and $e_{j}$ denote two arbitrary eigenvalues of $H^{\text {sc }}$ and $\mathcal{H}$, respectively. Although the spectra of both the scalar and the spin Calogero models can be easily computed, the previous formula cannot be directly used to compute the spectrum of the PF chain. Indeed, it is not obvious a priori which energies of $H$ and $H^{\text {sc }}$ can be combined to yield an energy of the PF chain. However, from Eq. (2.7) it is immediate to obtain the following exact relation between the partition functions $Z, Z^{\text {sc }}$ and $\mathcal{Z}$ of the Hamiltonians $H, H^{\text {sc }}$ and $\mathcal{H}$ :

$$
\mathcal{Z}(T)=\lim _{a \rightarrow \infty} \frac{Z(2 a T / J)}{Z^{\mathrm{sc}}(2 a T / J)} .
$$

This formula, first derived by Polychronakos [32], is the mainstay of the freezing trick method; see [39, 40] for a rigorous proof.

We shall next evaluate both the numerator and the denominator in the previous equation. To begin with, the partition function of the scalar Calogero model is easily computed [32], with the result

$$
Z^{\mathrm{sc}}(2 a T / J)=q^{\frac{J E_{g}}{2 a}} \prod_{i=1}^{N}\left(1-q^{i J}\right)^{-1},
$$

where

$$
E_{\mathrm{g}}=a^{2} N(N-1)+a N
$$

is the ground state energy.

Let us now turn to the partition function of $H$. Consider, to this end, the spin functions

$$
\psi_{\ell, \mathbf{s}}(\mathbf{x})=\rho(\mathbf{x}) \Lambda\left(\prod_{i=1}^{N} x_{i}^{\ell_{i}}\left|s_{1}, \ldots, s_{N}\right\rangle\right),
$$

where $\ell_{i} \in \mathbb{N} \cup\{0\}, \boldsymbol{\ell}=\left(\ell_{1}, \ldots, \ell_{N}\right), \mathbf{s}=\left(s_{1}, \ldots, s_{N}\right)$,

$$
\rho(\mathbf{x})=\mathrm{e}^{-a r^{2} / 2} \prod_{1 \leqslant i<j \leqslant N}\left|x_{i}-x_{j}\right|^{a}, \quad r^{2} \equiv \sum_{i=1}^{N} x_{i}^{2},
$$


is the ground state of the scalar Hamiltonian $H^{\text {sc }}$ and $\Lambda$ is the total symmetrizer with respect to particle permutations. The above states are a (non-orthonormal) basis of the Hilbert space of $H$ provided that (for instance) $\ell_{i} \geqslant \ell_{i+1}$ for all $i$ and $s_{i} \geqslant s_{i+1}$ whenever $\ell_{i}=\ell_{i+1}$. It was shown in Ref. [41] that $H_{0}$ acts triangularly on the latter basis, with eigenvalues

$$
E_{\mathrm{g}}+2 a|\boldsymbol{\ell}|, \quad|\boldsymbol{\ell}| \equiv \sum_{i=1}^{N} \ell_{i} .
$$

On the other hand, from the identities

$$
S_{i j} S_{i}^{\alpha}=S_{j}^{\alpha} S_{i j}, \quad S_{i j} S_{j}^{\alpha}=S_{i}^{\alpha} S_{i j} ; \quad S_{i j} S_{k}^{\alpha}=S_{k}^{\alpha} S_{i j}, \quad k \neq i, j,
$$

it immediately follows that the operators $S^{\alpha}$ commute with the spin permutation operators $S_{i j}$, and hence with $\Lambda$. By Eq. (1.3) we then have

$$
S^{\alpha} \psi_{\ell, \mathbf{s}}(\mathbf{x})=\rho(\mathbf{x}) \Lambda\left(\prod_{i=1}^{N} x_{i}^{\ell_{i}} S^{\alpha}\left|s_{1}, \ldots, s_{N}\right\rangle\right)=c^{\alpha}(\mathbf{s}) \psi_{\ell, \mathbf{s}},
$$

where the eigenvalue $c^{\alpha}(\mathbf{s})$ is given by

$$
c^{\alpha}(\mathbf{s})=\sum_{i=1}^{N}\left(\delta_{s_{i}}^{\alpha}-\delta_{s_{i}}^{m}\right) \equiv \#\left\{s_{i}=\alpha\right\}-\#\left\{s_{i}=m\right\} .
$$

In the previous formula, the symbol \#\{si $=k\}$ denotes the number of components of the vector $\mathbf{s}$ equal to $k$. It follows that the term $\sum_{\alpha=1}^{m-1} B_{\alpha} S^{\alpha}$ in Eq. 2.1] is diagonal in the basis 2.11, with eigenvalues

$$
\sum_{\alpha=1}^{m-1} c^{\alpha}(\mathbf{s}) B_{\alpha}=\sum_{\alpha=1}^{m-1} B_{\alpha} \#\left\{s_{i}=\alpha\right\}-\#\left\{s_{i}=m\right\} \sum_{\alpha=1}^{m-1} B_{\alpha} .
$$

In view of the latter equation, it is convenient to introduce the notation

$$
B_{m}=-\sum_{\alpha=1}^{m-1} B_{\alpha},
$$

so that $\sum_{\alpha=1}^{m} B_{\alpha}=0$. Using this notation we can rewrite the eigenvalue 2.13 in the more compact form

$$
\sum_{\alpha=1}^{m} B_{\alpha} \#\left\{s_{i}=\alpha\right\} \equiv \sum_{i=1}^{N} B_{s_{i}} .
$$

From the previous considerations and Eqs. 2.12)-(2.15) it then follows that the Hamiltonian 2.1] acts triangularly on the basis 2.11, with eigenvalues

$$
E_{\ell, \mathrm{s}}=E_{\mathrm{g}}+\frac{2 a}{J}\left(J|\boldsymbol{\ell}|-\sum_{i=1}^{N} B_{s_{i}}\right) .
$$

Using the previous equation, it is a straightforward matter to compute the partition function of the spin Calogero model 2.1). To this end, let us represent the multiindex $\boldsymbol{\ell}$ in Eq. 2.17) as

$$
\boldsymbol{\ell}=(\overbrace{\lambda_{1}, \ldots, \lambda_{1}}^{k_{1}}, \ldots, \overbrace{7}^{k_{r}, \ldots, \lambda_{r}}),
$$


with $\lambda_{1}>\lambda_{2}>\cdots>\lambda_{r} \geqslant 0$ and $\left(k_{1}, \ldots, k_{r}\right) \equiv \mathbf{k} \in \mathcal{P}_{N}$. We then have

$$
Z(2 a T / J)=q^{\frac{J E_{g}}{2 a}} \sum_{\mathbf{k} \in \mathcal{P}_{N}} \sum_{\lambda_{1}>\cdots>\lambda_{r} \geqslant 0} q^{\sum_{i=1}^{r} J k_{i} \lambda_{i}} \sum_{\mathbf{s} \in \ell} q^{-\sum_{i=1}^{N} B_{s_{i}}}
$$

where the last sum is extended to all spin quantum numbers $\mathbf{s}$ compatible with the multiindex $\ell$ given by Eq. (2.17), i.e., such that $s_{i} \geqslant s_{i+1}$ whenever $l_{i}=l_{i+1}$. In fact, since the latter sum clearly depends on $\boldsymbol{\ell}$ only through $\mathbf{k}$, from now on we denote it by $\Sigma(\mathbf{k})$. Clearly, by Eq. 2.17) we have

$$
\Sigma(\mathbf{k})=\prod_{i=1}^{r} \sum_{s_{1} \geqslant \cdots \geqslant s_{k_{i}}} q^{-\sum_{j=1}^{k_{i}} B_{s_{j}}}
$$

With this notation, Eq. 2.18] becomes

$$
Z(2 a T / J)=q^{\frac{J E_{g}}{2 a}} \sum_{\mathbf{k} \in \mathcal{P}_{N}} \Sigma(\mathbf{k}) \sum_{\lambda_{1}>\cdots>\lambda_{r} \geqslant 0} q^{\sum_{i=1}^{r} J k_{i} \lambda_{i}} .
$$

The inner sum in the RHS is easily computed by performing the change of indices $v_{i}=\lambda_{i}-\lambda_{i+1}$, where $i=1, \ldots, r$ and $\lambda_{r+1} \equiv 0$, since the new indices independently range from $1-\delta_{i r}$ to $\infty$. We thus obtain

$$
\sum_{\lambda_{1}>\cdots>\lambda_{r} \geqslant 0} q^{\sum_{i=1}^{r} J k_{i} \lambda_{i}}=\left(1-q^{N J}\right)^{-1} \prod_{i=1}^{r-1} \frac{q^{J K_{i}}}{1-q^{J K_{i}}},
$$

and therefore

$$
Z(2 a T / J)=q^{\frac{J E_{g}}{2 a}} \sum_{\mathbf{k} \in \mathcal{P}_{N}} \Sigma(\mathbf{k}) q^{\sum_{i=1}^{r-1} J K_{i}} \prod_{i=1}^{r}\left(1-q^{J K_{i}}\right)^{-1},
$$

where the positive integers $K_{i}$ were defined in Eq. (1.10). From the freezing trick relation (2.8) and Eqs. (2.9)-(2.21), we immediately obtain the following explicit expression for the partition function of the $\mathrm{PF}$ chain (1.1)-(1.7b) in the presence of an arbitrary su $(m)$ magnetic field:

$$
\mathcal{Z}(q)=\sum_{\mathbf{k} \in \mathcal{P}_{N}} \Sigma(\mathbf{k}) q^{\sum_{i=1}^{r-1} J K_{i}} \prod_{i=1}^{N-r}\left(1-q^{J K_{i}^{\prime}}\right) .
$$

The previous argument can be applied with minor modifications to both the HS and FI chains, thereby obtaining the following general expression for the partition function of the HS-type chains (1.1)-(1.7):

$$
\mathcal{Z}(q)=\sum_{\mathbf{k} \in \mathcal{P}_{N}} \Sigma(\mathbf{k}) q^{\Gamma_{i=1}^{r-1} J \mathcal{E}\left(K_{i}\right)} \prod_{i=1}^{N-r}\left(1-q^{J \mathcal{E}\left(K_{i}^{\prime}\right)}\right)
$$

Note that when the magnetic field vanishes, by Eq. (2.19) $\Sigma(\mathbf{k})$ becomes $\prod_{i=1}^{r}\left(\begin{array}{c}m+k_{i}-1 \\ k_{i}\end{array}\right)$, and the previous expression for $\mathcal{Z}$ reduces to its zero field version (1.9).

A few remarks on the function $\Sigma(\mathbf{k})$ are now in order. Let us first note that the latter function can be expressed as

$$
\Sigma(\mathbf{k})=\prod_{i=1}^{r} \sigma\left(k_{i}\right)
$$


where

$$
\sigma(k)=\sum_{s_{1} \geqslant \cdots \geqslant s_{k}} q^{-\sum_{j=1}^{k} B_{s_{j}}} .
$$

The function $\sigma(k)$ can be considerably simplified by noting that the summation indices $s_{1}, \ldots, s_{k}$ can be expressed as

$$
\overbrace{m, \ldots, m}^{p_{m}}, \overbrace{m-1, \ldots, m-1}^{p_{m-1}}, \ldots, \overbrace{1, \ldots, 1}^{p_{1}},
$$

with $\sum_{\alpha=1}^{m} p_{\alpha}=k$. Thus

$$
\sum_{j=1}^{k} B_{s_{j}}=\sum_{\alpha=1}^{m} p_{\alpha} B_{\alpha},
$$

and therefore

$$
\sigma(k)=\sum_{p_{1}+\cdots+p_{m}=k} \prod_{\alpha=1}^{m} q^{-p_{\alpha} B_{\alpha}} .
$$

The latter sum can be easily evaluated by noting that

$$
\sigma(k)=h_{k}\left(q^{-B_{1}}, \ldots, q^{-B_{m}}\right),
$$

where $h_{k}(\mathbf{x})$ denotes the complete homogeneous symmetric polynomial of degree $k$ in $m$ variables $\left(x_{1}, \ldots, x_{m}\right) \equiv \mathbf{x}$, given by

$$
h_{k}(\mathbf{x})=\sum_{p_{1}+\cdots+p_{m}=k} x_{1}^{p_{1}} \cdots x_{m}^{p_{m}} .
$$

Recalling that $h_{k}$ is the Schur polynomial associated with the single-row partition $(k)$ and using Jacobi's definition of the latter polynomials in terms of determinants [42] we conclude that

$$
\sigma(k)=\prod_{1 \leqslant i<j \leqslant m}\left(q^{-B_{i}}-q^{-B_{j}}\right)^{-1} \cdot\left|\begin{array}{ccc}
q^{-(k+m-1) B_{1}} & \ldots & q^{-(k+m-1) B_{m}} \\
q^{-(m-2) B_{1}} & \cdots & q^{-(m-2) B_{m}} \\
q^{-(m-3) B_{1}} & \cdots & q^{-(m-3) B_{m}} \\
\ldots & \ldots & \ldots \\
q^{-B_{1}} & \ldots & q^{-B_{m}} \\
1 & \cdots & 1
\end{array}\right| .
$$

Expanding the determinant along the first row we obtain the alternative expression

$$
\sigma(k)=\sum_{i=1}^{m} q^{-(k+m-1) B_{i}} \prod_{\substack{j=1 \\ j \neq i}}^{m}\left(q^{-B_{i}}-q^{-B_{j}}\right)^{-1} .
$$

In particular, for spin $1 / 2(m=2)$ we have $B_{1}=B=-B_{2}$, and either expression for $\sigma(k)$ easily yields

$$
\sigma(k)=\frac{q^{(k+1) B}-q^{-(k+1) B}}{q^{B}-q^{-B}}=\frac{\sinh ((k+1) \beta B)}{\sinh (\beta B)} .
$$

The RHS of the latter formula can be conveniently expressed using the $q$-number notation. Recall, to this end, that given two real numbers $x$ and $w>0$ the symmetric $w$-number $[[x]]_{w}$ is define by

$$
[[x]]_{w}=\frac{w^{\frac{x}{2}}-w^{-\frac{x}{2}}}{w^{\frac{1}{2}}-w^{-\frac{1}{2}}},
$$


which reduces to the ordinary number $x$ for $w=1$. We then have (in the spin $1 / 2$ case)

$$
\sigma(k)=[[k+1]]_{q^{2 B}},
$$

so that the partition function of the spin $1 / 2$ chains 1.1 - $(1.7)$ can be concisely written as

$$
\mathcal{Z}(q)=\sum_{\mathbf{k} \in \mathcal{P}_{N}} \prod_{i=1}^{r}\left[\left[k_{i}+1\right]\right]_{q^{2 B}} \cdot q^{\sum_{i=1}^{r-1} J \mathcal{E}\left(K_{i}\right)} \prod_{i=1}^{N-r}\left(1-q^{J \mathcal{E}\left(K_{i}^{\prime}\right)}\right) .
$$

Note, again, that in the absence of a magnetic field the previous expression obviously reduces to Eq. [1.9] with $m=2$.

\section{The associated vertex models}

In this section we shall prove that the spectrum of the HS chains (1.1)-(1.7) coincides with that of a classical inhomogeneous vertex model, which differs from the one in Eq. (1.12) by the addition of a magnetic field term. Our approach is based on the notion of generating function for the partition function, as used in Ref. [35] for the zero magnetic field case.

To this end, following the latter reference we define the generating function $\mathcal{F}$ of the zerofield partition function (1.9) as

$$
\mathcal{F}(\mathbf{x})=\sum_{\mathbf{k} \in \mathcal{P}_{N}} \prod_{i=1}^{r} h_{k_{i}}(\mathbf{x}) \cdot q^{\sum_{i=1}^{r-1} J \mathcal{E}\left(K_{i}\right)} \prod_{i=1}^{N-r}\left(1-q^{J \mathcal{E}\left(K_{i}^{\prime}\right)}\right) .
$$

Since

$$
h_{k}(1, \ldots, 1)=\left(\begin{array}{c}
m+k-1 \\
k
\end{array}\right),
$$

by Eq. (1.9) we have $\mathcal{Z}_{0}(q)=\mathcal{F}(1, \ldots, 1)$. More generally, substituting the expression (2.28) for $\sigma(k)$ into Eqs. 2.22)-(2.24) and using the definition of $\mathcal{F}(\mathbf{x})$ we readily obtain the identity

$$
\mathcal{Z}(q)=\mathcal{F}\left(q^{-B_{1}}, \ldots, q^{-B_{m}}\right) \text {. }
$$

Similarly, the generating function $\mathcal{F}^{\mathrm{v}}$ for the partition function of the classical vertex model 1.12 was defined in Ref. [35] by

$$
\mathcal{F}^{\mathrm{V}}(\mathbf{x})=\sum_{n_{1}, \ldots, n_{N}=0}^{m} x_{1}^{w_{1}(\mathbf{n})} \cdots x_{m}^{w_{m}(\mathbf{n})} q^{E_{0}(\mathbf{n})}
$$

where the nonnegative integers $w_{\alpha}(\mathbf{n})$ are given by

$$
w_{\alpha}(\mathbf{n})=\#\left\{n_{k}=\alpha\right\} .
$$

Again, it is obvious that the partition function $Z_{0}^{\mathrm{V}}$ of the model (1.12)-(1.13) is the value of its generating function at the point $(1, \ldots, 1)$. One of the fundamental results in Ref. [35] is the fact that the generating functions (3.1) and (3.3) are identically equal, i.e.,

$$
\mathcal{F}(\mathbf{x})=\mathcal{F}^{\mathrm{V}}(\mathbf{x}), \quad \forall \mathbf{x} \in \mathbb{R}^{m} .
$$


Evaluating the previous identity at the point $(1, \ldots, 1)$ we obtain the equality of the zero-field partition functions $\mathcal{Z}_{0}$ and $\mathcal{Z}_{0}^{\mathrm{V}}$, which is in fact the main result in Ref. [35]. On the other hand, if we evaluate the identity (3.4) at the point $\left(q^{-B_{1}}, \ldots, q^{-B_{m}}\right)$ and use Eq. (3.2) we immediately obtain

$$
\mathcal{Z}(q)=\mathcal{F}^{\mathrm{V}}\left(q^{-B_{1}}, \ldots, q^{-B_{m}}\right)=\sum_{n_{1}, \ldots, n_{N}=0}^{m} q^{-\sum_{\alpha=1}^{m} B_{\alpha} w_{\alpha}(\mathbf{n})} q^{E_{0}(\mathbf{n})} .
$$

Taking into account that

$$
\sum_{\alpha=1}^{m} B_{\alpha} w_{\alpha}(\mathbf{n})=\sum_{i=1}^{N} B_{n_{i}}
$$

we can rewrite Eq. 3.5 as

$$
\mathcal{Z}(q)=\sum_{n_{1}, \ldots, n_{N}=0}^{m} q^{E_{0}(\mathbf{n})-\sum_{i=1}^{N} B_{n_{i}}} \equiv \mathcal{Z}^{\mathrm{V}}(q)
$$

where $\mathcal{Z}^{\mathrm{V}}$ denotes the partition function of the inhomogeneous classical vertex model with energies

$$
E(\mathbf{n})=E_{0}(\mathbf{n})-\sum_{i=1}^{N} B_{n_{i}}, \quad n_{k} \in\{1, \ldots, m\},
$$

with $E_{0}(\mathbf{n})$ given by Eqs. (1.12)-(1.13). Therefore, as stated at the beginning of this section, the spectrum of the spin chains $(1.1)-(1.7)$ is identical to that of the classical vertex models defined by Eq. (1.12)-3.7).

In the spin $1 / 2$ case, the previous equation can be simplified by noting that $\delta(j, k)$ in Eq. 1.13 can be expressed as

$$
\delta(j, k)=(j-1)(2-k), \quad j, k=1,2,
$$

and similarly

$$
B_{j}=(3-2 j) B, \quad j=1,2 .
$$

Introducing the spin variables $\sigma_{k}=3-2 n_{k}$, Eq. 3.7 becomes

$$
E(\sigma)=\frac{J}{4} \sum_{i=1}^{N-1} \mathcal{E}(i)\left(1-\sigma_{i}\right)\left(1+\sigma_{i+1}\right)-B \sum_{1=1}^{N} \sigma_{i}, \quad \sigma_{k} \in\{ \pm 1\}
$$

The latter equation can be alternatively written as

$$
E(\sigma)=-\frac{J}{4} \sum_{i=1}^{N-1} \mathcal{E}(i)\left(\sigma_{i} \sigma_{i+1}-1\right)-\sum_{i=1}^{N} \mathcal{B}(i) \sigma_{i},
$$

where

$$
\mathcal{B}(i) \equiv B+\frac{J}{4}[\mathcal{E}(i)-\mathcal{E}(i-1)]
$$

and we have set $\mathcal{E}(0)=\mathcal{E}(N)=0$. The last two equations define a classical inhomogeneous Ising model in one dimension, where the coupling between the spins $i$ and $i+1$ is proportional to the dispersion relation $\mathcal{E}(i)$, and the external magnetic field (also inhomogeneous) is given by Eq. (3.12). 


\section{Thermodynamics of the spin $1 / 2$ chains}

We shall next take advantage of the representation 3.10 of the spectrum of the HS-type chains (1.1)-(1.7) with spin $1 / 2$ in order to determine their equilibrium thermodynamics in a unified way. To this end, we must first normalize the Hamiltonian (1.6) to ensure that the energy per spin is finite (and nonzero) in the thermodynamic limit. It is well known that when $N \gg 1$ the mean energy of the HS-type chains is $\mathrm{O}\left(N^{r}\right)$, with $r=2$ for the PF chain and $r=3$ for the HS and FI chains [19, 33, 34]. Hence from now on we shall take

$$
J= \begin{cases}K / N^{2}, & \text { for the HS and FI chains } \\ K / N, & \text { for the PF chain, }\end{cases}
$$

where the constant $K$ is independent of $N$. With this proviso, Eq. 3.10) can be rewritten in terms of the discrete variables $x_{i} \equiv i / N \in(0,1)$ as

$$
E(\boldsymbol{\sigma})=\frac{K}{4} \sum_{i=1}^{N-1} \varepsilon_{i}\left(1-\sigma_{i}\right)\left(1+\sigma_{i+1}\right)-B \sum_{i=1}^{N} \sigma_{i},
$$

where

$$
\varepsilon_{i}= \begin{cases}x_{i}\left(1-x_{i}\right), & \text { for the HS chain } \\ x_{i}, & \text { for the PF chain } \\ x_{i}\left(\gamma_{N}+x_{i}\right), & \text { for the FI chain, }\end{cases}
$$

and $\gamma_{N} \equiv(\alpha-1) / N$. We shall further assume that $\gamma_{N}$ has a finite limit $\gamma \geqslant 0$ as $N \rightarrow \infty$. With the above notation, the partition function of the chains $1.6 p-(1.7)$ can be collectively expressed as

$$
\mathcal{Z}(q)=\sum_{\sigma} q^{d\left(\sigma_{1}, \sigma_{2}\right) \varepsilon_{1}-\frac{B}{2}\left(\sigma_{1}+\sigma_{2}\right)} \cdots q^{d\left(\sigma_{N-1}, \sigma_{N}\right) \varepsilon_{N-1}-\frac{B}{2}\left(\sigma_{N-1}+\sigma_{N}\right)} q^{-\frac{B}{2}\left(\sigma_{1}+\sigma_{N}\right)},
$$

where

$$
d\left(\sigma, \sigma^{\prime}\right)=\frac{K}{4}(1-\sigma)\left(1+\sigma^{\prime}\right) .
$$

Equation 4.4] can be more concisely written as

$$
\mathcal{Z}(q)=\operatorname{tr}\left(U T_{1} \cdots T_{N-1}\right),
$$

where $U$ and $T_{i}$ are $2 \times 2$ matrices with elements

$$
U_{\sigma \sigma^{\prime}}=q^{-\frac{B}{2}\left(\sigma+\sigma^{\prime}\right)}, \quad\left(T_{i}\right)_{\sigma \sigma^{\prime}}=q^{d\left(\sigma, \sigma^{\prime}\right) \varepsilon_{i}-\frac{B}{2}\left(\sigma+\sigma^{\prime}\right)} ; \quad \sigma, \sigma^{\prime}= \pm 1,
$$

or equivalently,

$$
U=\left(\begin{array}{cc}
q^{B} & 1 \\
1 & q^{-B}
\end{array}\right), \quad T_{i}=\left(\begin{array}{cc}
q^{B} & q^{K \varepsilon_{i}} \\
1 & q^{-B}
\end{array}\right) .
$$

The transfer matrix $T_{i}$ has two distinct eigenvalues

$$
\lambda_{i, \pm}=\cosh (\beta B) \pm \sqrt{\sinh ^{2}(\beta B)+\mathrm{e}^{-K \beta \varepsilon_{i}}},
$$

and is therefore diagonalizable. A straightforward calculation shows that

$$
T_{i}=P_{i} D_{i} P_{i}^{-1},
$$


where

$$
D_{i}=\left(\begin{array}{cc}
\lambda_{i,+} & 0 \\
0 & \lambda_{i,-}
\end{array}\right), \quad P_{i}=\left(\begin{array}{cc}
-a+r_{i} & -a-r_{i} \\
1 & 1
\end{array}\right),
$$

and we have set

$$
a=\sinh (\beta B), \quad r_{i}=\sqrt{\sinh (\beta B)^{2}+\mathrm{e}^{-K \beta \varepsilon_{i}}} .
$$

We thus have

$$
T_{1} \cdots T_{N-1}=P_{1}\left(D_{1} C_{1}\right) \cdots\left(D_{N-2} C_{N-2}\right) D_{N-1} P_{N-1}^{-1},
$$

where $C_{i} \equiv P_{i}^{-1} P_{i+1}$ is a symmetric matrix explicitly given by

$$
C_{i}=\frac{1}{2 r_{i}}\left(\begin{array}{cc}
r_{i}+r_{i+1} & r_{i}-r_{i+1} \\
r_{i}-r_{i+1} & r_{i}+r_{i+1}
\end{array}\right) \text {. }
$$

The key observation at this point is that

$$
C_{i}=I+\mathrm{O}\left(N^{-1}\right)
$$

as $N \rightarrow \infty$. Indeed, note first of all that

$$
0 \leqslant \varepsilon_{i} \leqslant \varepsilon_{\max },
$$

where

$$
\varepsilon_{\max }= \begin{cases}\frac{1}{4}, & \text { for the HS chain } \\ 1, & \text { for the PF chain } \\ \gamma_{N}+1, & \text { for the FI chain, }\end{cases}
$$

so that $\varepsilon_{i}=\mathrm{O}(1)$. On the other hand, since $0<x_{i}<1$ the difference

$$
\varepsilon_{i+1}-\varepsilon_{i}= \begin{cases}\frac{1}{N}\left(1-x_{i}-x_{i+1}\right), & \text { for the HS chain } \\ \frac{1}{N}, & \text { for the PF chain } \\ \frac{1}{N}\left(\gamma_{N}+x_{i}+x_{i+1}\right), & \text { for the FI chain }\end{cases}
$$

is $\mathrm{O}\left(N^{-1}\right)$, and hence

$$
\frac{r_{i+1}}{r_{i}}=1+\mathrm{O}\left(N^{-1}\right)
$$

which establishes our claim.

From Eq. 4.13), it follows that when $N \rightarrow \infty$ the leading term of $\mathcal{Z}(q)$ is given by

$$
\operatorname{tr}\left(P_{N-1}^{-1} U P_{1} D_{1} \cdots D_{N-1}\right)=\operatorname{tr}\left[P_{N-1}^{-1} U P_{1}\left(\begin{array}{cc}
\lambda_{+} & 0 \\
0 & \lambda_{-}
\end{array}\right)\right],
$$

where

$$
\lambda_{ \pm} \equiv \prod_{i=1}^{N-1} \lambda_{i, \pm}
$$

Note also that

$$
\lim _{N \rightarrow \infty} \varepsilon_{1}=0, \quad \lim _{N \rightarrow \infty} \varepsilon_{N-1}=\varepsilon_{\infty} \equiv \begin{cases}0, & \text { for the HS chain } \\ 1, & \text { for the PF chain } \\ \gamma+1, & \text { for the FI chain, }\end{cases}
$$


so that the matrices $P_{1}$ and $P_{N-1}$ both have finite limits as $N \rightarrow \infty$. Calling

$$
r_{\infty}=\sqrt{\sinh ^{2}(\beta B)+\mathrm{e}^{-K \beta \varepsilon_{\infty}}}
$$

we then have

$$
\begin{aligned}
& \lim _{N \rightarrow \infty} P_{N-1}^{-1} U P_{1}=\frac{1}{2 r_{\infty}}\left(\begin{array}{cc}
1 & r_{\infty}+a \\
-1 & r_{\infty}-a
\end{array}\right)\left(\begin{array}{cc}
\mathrm{e}^{-\beta B} & 1 \\
1 & \mathrm{e}^{\beta B}
\end{array}\right)\left(\begin{array}{cc}
\mathrm{e}^{-\beta B} & -\mathrm{e}^{\beta B} \\
1 & 1
\end{array}\right) \\
& =\frac{\cosh (\beta B)}{r_{\infty}}\left(\begin{array}{cc}
r_{\infty}+\cosh (\beta B) & 0 \\
r_{\infty}-\cosh (\beta B) & 0
\end{array}\right) \text {, }
\end{aligned}
$$

and therefore

$$
\operatorname{tr}\left[P_{N-1}^{-1} U P_{1}\left(\begin{array}{cc}
\lambda_{+} & 0 \\
0 & \lambda_{-}
\end{array}\right)\right]=\cosh (\beta B)\left(1+\frac{\cosh (\beta B)}{r_{\infty}}\right) \lambda_{+}
$$

is the leading term of $\mathcal{Z}(q)$ as $N \rightarrow \infty$. Since the first two factors in the RHS of this formula are independent of $N$, in the thermodynamic limit the free energy per spin $f(B, T)$ is simply given by

$$
\begin{aligned}
f(B, T) & =-\lim _{N \rightarrow \infty} \frac{\log \mathcal{Z}}{N \beta}=-\frac{1}{\beta} \lim _{N \rightarrow \infty} N^{-1} \sum_{i=1}^{N-1} \log \lambda_{i,+} \\
& =-\frac{1}{\beta} \lim _{N \rightarrow \infty} N^{-1} \sum_{i=1}^{N-1} \log \left(\cosh (\beta B)+\sqrt{\sinh ^{2}(\beta B)+\mathrm{e}^{-K \beta \varepsilon\left(x_{i}\right)}}\right),
\end{aligned}
$$

where the dispersion function $\varepsilon(x)$ is defined by

$$
\varepsilon(x)= \begin{cases}x(1-x), & \text { for the HS chain } \\ x, & \text { for the PF chain } \\ x(\gamma+x), & \text { for the FI chain }\end{cases}
$$

(cf. Eq. (4.3). We thus obtain the following remarkable formula for the free energy per site of the HS-type chains (1.6)-(1.7):

$$
f(B, T)=-\frac{1}{\beta} \int_{0}^{1} \log \left(\cosh (\beta B)+\sqrt{\sinh ^{2}(\beta B)+\mathrm{e}^{-K \beta \varepsilon(x)}}\right) \mathrm{d} x .
$$

From the latter equation it is immediate to obtain the magnetization per site:

$$
\mu(B, T)=-\frac{\partial f(B, T)}{\partial B}=\int_{0}^{1} \frac{\sinh (\beta B)}{\sqrt{\sinh ^{2}(\beta B)+\mathrm{e}^{-K \beta \varepsilon(x)}}} \mathrm{d} x .
$$

For finite temperature, the magnetic susceptibility is also easily computed from the previous formula:

$$
\chi(B, T)=\frac{\partial \mu(B, T)}{\partial B}=\beta \int_{0}^{1} \frac{\cosh (\beta B) \mathrm{e}^{-K \beta \varepsilon(x)}}{\left(\sinh ^{2}(\beta B)+\mathrm{e}^{-K \beta \varepsilon(x)}\right)^{3 / 2}} \mathrm{~d} x .
$$

In the case of the FI chain, some of the above equations have previously appeared in Ref. [6]. More precisely, Eq. (4.18) with $B=0$ reduces to Eq. (21) of the latter reference, while Eq. 4.19) 
for $K>0$ essentially coincides with Eq. (24) of Ref. [6], which is stated without proof. As first noted in this reference for the $B=0$ case, Eqs. (4.18)-(4.20) imply that the HS chain is thermodynamically equivalent to an FI chain with $\gamma=-1$ and $K \rightarrow-K$.

The free energy and magnetization per spin (4.18)-(4.19) admit a simpler expression in terms of the so-called dressed energy $\varepsilon_{\mathrm{dr}}(x)$ defined by

$$
\sinh \left(\beta \varepsilon_{\mathrm{dr}}(x)\right)=\mathrm{e}^{\frac{K \beta}{2} \varepsilon(x)} \sinh (\beta B),
$$

namely,

$$
\begin{aligned}
& f(B, T)=-\frac{1}{\beta} \int_{0}^{1} \log \left(\frac{\sinh \left[\beta\left(\varepsilon_{\mathrm{dr}}(x)+B\right)\right]}{\sinh \left[\beta \varepsilon_{\mathrm{dr}}(x)\right]}\right) \mathrm{d} x \\
& \mu(B, T)=\int_{0}^{1} \tanh \left(\beta \varepsilon_{\mathrm{dr}}(x)\right) \mathrm{d} x .
\end{aligned}
$$

These are the well-known expressions for the free energy (up to an additive constant depending on the normalization of $\mathcal{H}$ ) and magnetization per particle of a system of free bosons with oneparticle energies $\varepsilon(x), x \in[0,1]$, in the presence of a constant magnetic field $B$. Note in this respect that, in contrast with the dispersion relation $\varepsilon(x)$, the dressed energy $\varepsilon_{\mathrm{dr}}(x)$ depends on $\beta$, $B$ and $K$. Note also that in the case of the HS chain Eq. (4.22) is essentially equivalent to Eq. (37) in Ref. [43], deduced by means of Haldane's spinon gas approach [23].

For future reference, we shall also compute the internal energy and entropy per spin. To begin with, the internal energy per spin $u$ is given by

$$
u(B, T)=\frac{\partial}{\partial \beta}(\beta f(B, T))=\frac{1}{2} \int_{0}^{1}\left(\frac{K \varepsilon(x) \mathrm{e}^{-K \beta \varepsilon(x)}}{\cosh (\beta B)+r(x)}-2 B \sinh (\beta B)\right) \frac{\mathrm{d} x}{r(x)},
$$

with

$$
r(x)=\sqrt{\sinh ^{2}(\beta B)+\mathrm{e}^{-K \beta \varepsilon(x)}} .
$$

The entropy per spin $s$ is then easily derived from the usual formula

$$
s(B, T)=\frac{u(B, T)-f(B, T)}{T} .
$$

\subsection{The PF chain}

We have not been able to express the thermodynamic functions $4.18-4.25$ in terms of known special functions for arbitrary values of $B$ and $T$, with the remarkable exception of the Polychronakos-Frahm chain. Indeed, we shall now show that in this case the free and the internal energy, the entropy (and the specific heat) can be expressed in terms of the dilogarithm function [44]

$$
\operatorname{Li}_{2}(z)=-\int_{0}^{z} \frac{\log (1-t)}{t} \mathrm{~d} t, \quad z \in \mathbb{C} \backslash(1, \infty),
$$

while the magnetization and susceptibility are elementary functions of $B$ and $T$. In Eq. 4.26$] \log$ denotes the principal value of the $\operatorname{logarithm}$ (i.e., $-\pi<\operatorname{Im} \log z<\pi$ ), and the integral is taken along any path joining the origin with the point $z$ that does not intersect the branch cut $(1, \infty)$. 
We start by performing the change of variable $t^{2}=a^{2}+\mathrm{e}^{-\kappa x}$, where $a=\sinh (\beta B)$ and $\kappa=K \beta$, in Eq. $(4.18)$ for the free energy. We readily obtain

$$
K \beta^{2} f(B, T)=\int_{c}^{\sqrt{a^{2}+\mathrm{e}^{-\kappa}}}\left(\frac{1}{t+a}+\frac{1}{t-a}\right) \log (t+c) \mathrm{d} t,
$$

where $c=\cosh (\beta B)$. The linear change of variable

$$
z=-\frac{t \pm a}{c \mp a} \equiv-\mathrm{e}^{ \pm \beta B}(t \pm a)
$$

makes it possible to evaluate each of the last two integrals in closed form:

$$
\begin{aligned}
\int_{c}^{\sqrt{a^{2}+\mathrm{e}^{-\kappa}}} \frac{\log (t+c)}{t \pm a} \mathrm{~d} t= & \int_{-\mathrm{e}^{ \pm 2 \beta B}}^{-\mathrm{e}^{ \pm \beta B}\left( \pm a+\sqrt{\left.a^{2}+\mathrm{e}^{-\kappa}\right)}\right.}\left(\frac{\log (1-z)}{z} \mp \frac{\beta B}{z}\right) \mathrm{d} z \\
= & \operatorname{Li}_{2}\left(-\mathrm{e}^{ \pm 2 \beta B}\right)-\operatorname{Li}_{2}\left(-\mathrm{e}^{ \pm \beta B}\left( \pm a+\sqrt{\left.a^{2}+\mathrm{e}^{-\kappa}\right)}\right)\right. \\
& \mp \beta B \log \left( \pm a+\sqrt{a^{2}+\mathrm{e}^{-\kappa}}\right)+\beta^{2} B^{2} .
\end{aligned}
$$

Taking into account the dilogarithm identity [44]

$$
\operatorname{Li}_{2}\left(-\mathrm{e}^{x}\right)+\operatorname{Li}_{2}\left(-\mathrm{e}^{-x}\right)=-\frac{\pi^{2}}{6}-\frac{x^{2}}{2},
$$

after some straightforward algebra we finally obtain the following closed formula for the free energy of the PF chain:

$$
\begin{aligned}
-K \beta^{2}(f(B, T)+B)= & \frac{\pi^{2}}{6}+2 \beta B \log \left(\sinh (\beta B)+\sqrt{\sinh ^{2}(\beta B)+\mathrm{e}^{-K \beta}}\right) \\
& +\operatorname{Li}_{2}\left(-\mathrm{e}^{\beta B}\left[\sinh (\beta B)+\sqrt{\sinh ^{2}(\beta B)+\mathrm{e}^{-K \beta}}\right]\right) \\
& +\operatorname{Li}_{2}\left(-\mathrm{e}^{-\beta B}\left[-\sinh (\beta B)+\sqrt{\sinh ^{2}(\beta B)+\mathrm{e}^{-K \beta}}\right]\right) .
\end{aligned}
$$

The previous formula generalizes the analogous expression for zero magnetic field in Eq. (23) of Ref. [5]. Equation (4.28) can be somewhat simplified by expressing the RHS in terms of the dressed energy. Indeed, by Eq. (4.21) with $\varepsilon(x)=x$ we have

$$
\pm \sinh (\beta B)+\sqrt{\sinh ^{2}(\beta B)+\mathrm{e}^{-K \beta}}=\mathrm{e}^{\beta\left(-\frac{K}{2} \pm \varepsilon_{\mathrm{dr}}(1)\right)},
$$

and therefore

$$
-K \beta^{2} f(B, T)=\frac{\pi^{2}}{6}+2 \beta^{2} B \varepsilon_{\mathrm{dr}}(1)+\operatorname{Li}_{2}\left(-\mathrm{e}^{\beta\left(B+\varepsilon_{\mathrm{dr}}(1)-\frac{K}{2}\right)}\right)+\operatorname{Li}_{2}\left(-\mathrm{e}^{-\beta\left(B+\varepsilon_{\mathrm{dr}}(1)+\frac{K}{2}\right)}\right) .
$$

From the usual formulas for the internal energy, the entropy and the specific heat (per site)

$$
c(B, T)=\frac{\partial u(B, T)}{\partial T}
$$

in terms of the free energy, it immediately follows that these functions necessarily include a term proportional to the last two lines in Eq. (4.28), and therefore cannot be expressed purely 
in terms of elementary functions. On the other hand, since the magnetization is obtained from the free energy by differentiating with respect to the magnetic field $B$, it is clear from Eq. (4.28) that $\mu$ is an elementary function of $B$ and $T$. Differentiating Eq. (4.28) with respect to $B$, or simply performing the change of variables $t^{2}=a^{2}+\mathrm{e}^{-K \beta x}$ in Eq. 4.19), after a straightforward calculation we obtain the remarkable formula

$$
\mu(B, T)=1+\frac{2}{K \beta}\left[\log \left(\sinh (\beta B)+\sqrt{\sinh ^{2}(\beta B)+\mathrm{e}^{-K \beta}}\right)-\beta B\right] .
$$

Using Eq. 4.29, we can rewrite the previous formula in terms of the dressed energy as

$$
\mu(B, T)=\frac{2}{K}\left(\varepsilon_{\mathrm{dr}}(1)-B\right) .
$$

Differentiating the previous expressions for $\mu$ we also obtain the following closed formulas for the susceptibility per site:

$$
\chi(B, T)=\frac{2}{K}\left(\frac{\cosh (\beta B)}{\sqrt{\sinh ^{2}(\beta B)+\mathrm{e}^{-K \beta}}}-1\right)=\frac{2}{K}\left(\frac{\tanh \left(\beta \varepsilon_{\mathrm{dr}}(1)\right)}{\tanh (\beta B)}-1\right) .
$$

\section{The zero field case}

It is of interest to study the limit of the thermodynamic functions computed in the previous section for both $B \rightarrow 0$ and $T \rightarrow 0$. We shall deal in this section with the first of these limits, leaving the analysis of the zero temperature limit for the next one.

We shall start with the free energy and the internal energy per site, which can be immediately obtained by setting $B=0$ in Eqs. 4.18) and 4.24):

$$
\begin{aligned}
& f(0, T)=-\frac{1}{\beta} \int_{0}^{1} \log \left(1+\mathrm{e}^{-\frac{K \beta}{2} \varepsilon(x)}\right) \mathrm{d} x, \\
& u(0, T)=\frac{K}{2} \int_{0}^{1} \frac{\varepsilon(x)}{1+\mathrm{e}^{\frac{K \beta}{2} \varepsilon(x)}} \mathrm{d} x .
\end{aligned}
$$

From the above formulas and Eq. 4.25) it follows that the entropy per spin is given by

$$
\begin{aligned}
\frac{s(0, T)}{k_{\mathrm{B}}} & =\int_{0}^{1}\left[\log \left(1+\mathrm{e}^{-\frac{K \beta}{2} \varepsilon(x)}\right)+\frac{1}{2} \frac{K \beta \varepsilon(x)}{1+\mathrm{e}^{\frac{K \beta}{2} \varepsilon(x)}}\right] \mathrm{d} x \\
& =\int_{0}^{1}\left\{\log \left[2 \cosh \left(\frac{K \beta}{4} \varepsilon(x)\right)\right]-\frac{K \beta \varepsilon(x)}{4} \tanh \left(\frac{K \beta}{4} \varepsilon(x)\right)\right\} \mathrm{d} x .
\end{aligned}
$$

In the case of the HS chain, the latter equation is essentially equivalent to Eq. (15) in Ref. [23], which was deduced using the equivalence of the HS chain to an ideal gas of spinons. The zerofield specific heat

$$
c(0, T) \equiv \frac{\partial u(0, T)}{\partial T}
$$

can also be easily computed from Eq. 5.2, with the result

$$
\frac{c(0, T)}{k_{\mathrm{B}}}=\left(\frac{K \beta}{4}\right)^{2} \int_{0}^{1} \varepsilon_{17}^{2}(x) \operatorname{sech}^{2}\left(\frac{K \beta}{4} \varepsilon(x)\right) \mathrm{d} x .
$$


Obviously, the zero-field magnetization vanishes (cf. (4.19)), while Eq. 4.20) for the susceptibility reduces to

$$
\chi(0, T)=\beta \int_{0}^{1} \mathrm{e}^{\frac{K \beta}{2} \varepsilon(x)} \mathrm{d} x .
$$

In fact, the zero-field susceptibility can be expressed in terms of standard special functions for all spin chains of HS type. For the PF chain, we have already obtained a formula for $\chi$ in terms of elementary functions for all values of $B$ and $T$, cf. Eq. 44.32 . For the HS chain, a straightforward calculation shows that

$$
\chi(0, T)=\sqrt{\frac{2 \pi \beta}{|K|}} \mathrm{e}^{K \beta / 8} \times \begin{cases}\operatorname{erf}\left(\sqrt{\frac{K \beta}{8}}\right), & \mathrm{K}_{\dot{\iota} 0} \\ \operatorname{erf}\left(\sqrt{\frac{\mid K \beta}{8}}\right), & \mathrm{K}_{i} 0\end{cases}
$$

where

$$
\operatorname{erf}(z)=\frac{2}{\sqrt{\pi}} \int_{0}^{z} \mathrm{e}^{-t^{2}} \mathrm{~d} t, \quad \operatorname{erfi}(z)=\frac{2}{\sqrt{\pi}} \int_{0}^{z} \mathrm{e}^{t^{2}} \mathrm{~d} t=-\mathrm{i} \operatorname{erf}(\mathrm{i} z)
$$

denote respectively the error and the imaginary error functions [45]. Similarly, for the FI chain we have

$$
\chi(0, T)=\sqrt{\frac{\pi \beta}{2|K|}} \mathrm{e}^{-K \beta \gamma^{2} / 8} \times \begin{cases}\operatorname{erfi}\left((\gamma+2) \sqrt{\frac{K \beta}{8}}\right)-\operatorname{erfi}\left(\gamma \sqrt{\frac{K \beta}{8}}\right), & K>0 \\ \operatorname{erf}\left((\gamma+2) \sqrt{\frac{\mid K \beta}{8}}\right)-\operatorname{erf}\left(\gamma \sqrt{\frac{\mid K \beta}{8}}\right), \quad K<0 .\end{cases}
$$

From Eqs. (4.32), (5.6), and (5.7), and the well-known asymptotic formula [45]

$$
\operatorname{erfi} z \sim \frac{\mathrm{e}^{z^{2}}}{\sqrt{\pi} z} ; \quad|z| \rightarrow \infty, \quad|\arg z|<\frac{\pi}{4},
$$

it follows that when $K>0$ and $T \rightarrow 0$ we have

$$
\chi(0, T) \simeq \begin{cases}\sqrt{\frac{2 \pi \beta}{K}} \mathrm{e}^{K \beta / 8}, & \text { for the HS chain } \\ \frac{2}{K} \mathrm{e}^{K \beta / 2}, & \text { for the PF chain } \\ \frac{2}{(\gamma+2) K} \mathrm{e}^{(\gamma+1) K \beta / 2}, & \text { for the FI chain } .\end{cases}
$$

Again, in the case of the HS chain the previous asymptotic formula was first obtained by Haldane in Ref. [23]. From the latter equation it is apparent that the ferromagnetic zero-field susceptibility (and hence the correlation length) is exponentially divergent as $T \rightarrow 0$. This shows that all ferromagnetic chains of HS type undergo a phase transition at $T=B=0$, which we shall analyze in detail in the next section.

Equations (5.3) and (5.4) imply that the zero-field entropy and specific heat are even functions of the coupling constant $K$. This fact, which is not obvious a priori, was first noted by Haldane [23] for the entropy of the original HS chain. Haldane also pointed out that (with an appropriate choice of the ground state energy, which amounts to replacing $1-S_{i j}$ by $S_{i j}$ in 
Eq. (1.6) the internal energy of the HS chain is again even in $K$. One can easily show that this property is shared by all spin chains of HS type, since from Eq. 5.2] it readily follows that

$$
u(0, T ; K)-u(0, T ;-K)=\frac{K}{2} \int_{0}^{1} \varepsilon(x) \mathrm{d} x \equiv \frac{K \varepsilon_{0}}{2},
$$

with

$$
\varepsilon_{0}= \begin{cases}\frac{1}{6}, & \text { for the HS chain } \\ \frac{1}{2}, & \text { for the PF chain } \\ \frac{1}{6}(3 \gamma+2), & \text { for the FI chain. }\end{cases}
$$

In other words, $u(0, T)-\frac{K \varepsilon_{0}}{4}$ is an even function of $K$, which can be seen to be equivalent to Haldane's statement given our normalization of $\mathcal{H}$. A similar property, proved in exactly the same way, also holds for the free energy $f(0, T)$.

As we saw in the previous section, in the particular case of the Polychronakos-Frahm chain the thermodynamic functions $(5.1)-5.4$ ) can be expressed in closed form in terms of the dilogarithm function. Indeed, setting $B=0$ in Eq. (4.28) we obtain the simple formula

$$
f(0, T)=-\frac{2}{K \beta^{2}}\left(\operatorname{Li}_{2}\left(-\mathrm{e}^{-K \beta / 2}\right)+\frac{\pi^{2}}{12}\right) .
$$

From the latter equation it is straightforward to derive similar expressions for the internal energy, the entropy and the specific heat of the PF chain in the absence of a magnetic field, namely

$$
\begin{aligned}
& u(0, T)=\frac{2}{K \beta^{2}}\left(\operatorname{Li}_{2}\left(-\mathrm{e}^{-K \beta / 2}\right)+\frac{\pi^{2}}{12}\right)-\frac{1}{\beta} \log \left(1+\mathrm{e}^{-K \beta / 2}\right) \\
& \frac{s(0, T)}{k_{\mathrm{B}}}=\frac{4}{K \beta}\left(\operatorname{Li}_{2}\left(-\mathrm{e}^{-K \beta / 2}\right)+\frac{\pi^{2}}{12}\right)-\log \left(1+\mathrm{e}^{-K \beta / 2}\right) \\
& \frac{c(0, T)}{k_{\mathrm{B}}}=\frac{4}{K \beta}\left(\operatorname{Li}_{2}\left(-\mathrm{e}^{-K \beta / 2}\right)+\frac{\pi^{2}}{12}\right)-2 \log \left(1+\mathrm{e}^{-K \beta / 2}\right)-\frac{K \beta}{2\left(1+\mathrm{e}^{K \beta / 2}\right)} .
\end{aligned}
$$

\subsection{Discussion}

Using the previous explicit formulas, we have plotted the thermodynamic functions of the PF chain for zero magnetic field $5.9-5.12]$ in terms of the dimensionless temperature $\tau=(|K| \beta)^{-1}$; see Fig. 1 .

It is immediately apparent the qualitative similarity of these plots with the corresponding ones for a two-level system like, e.g., a paramagnetic spin $1 / 2$ ion or the one-dimensional Ising model with zero magnetic field [46]. In particular, the specific heat $c(0, T)$ clearly exhibits the so-called Schottky anomaly, characteristic of this type of systems. The Schottky peak, which can be numerically computed without difficulty from Eq. [5.12), is located at $|K| \beta \simeq 0.127762$, with a maximum value of $c(0, T)$ approximately equal to $0.439229 k_{\mathrm{B}}$. On the other hand, the low temperature behavior of $s(0, T)$ and $c(0, T)$ markedly differs from that of a two-level system.

\footnotetext{
${ }^{4}$ For the latter model, the effective half-gap is equal to the coupling between the spins.
} 

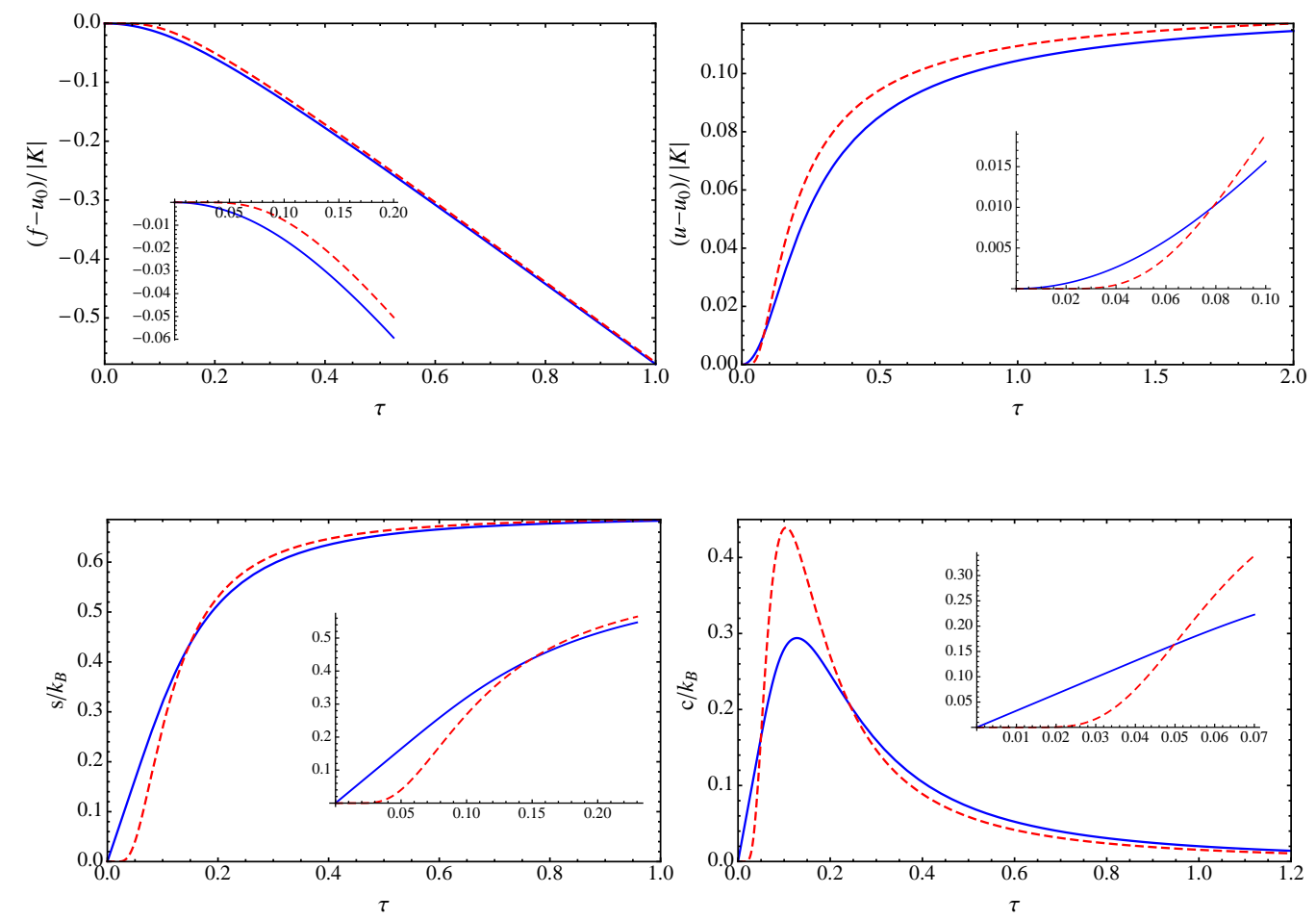

Figure 1: Free and internal energy, entropy, and specific heat (all of them per site) of the PF chain with zero magnetic field (solid blue line) and of a two-level system with half-gap $\Delta=|K| / 8$ (dashed red line) as functions of the dimensionless temperature $\tau=(|K| \beta)^{-1}$. In the first two plots, $u_{0}=\frac{K \varepsilon_{0}}{4}(1-\operatorname{sgn} K)$ denotes the common value of $u(0,0)$ and $f(0,0)$, cf. Eq. [5.17). Insets: low-temperature behavior of each of the above thermodynamic functions.

Indeed, from Eqs. (5.11) and (5.12) it immediately follows that

$$
\begin{array}{ll}
\frac{s(0, T)}{k_{\mathrm{B}}}=\frac{\pi^{2}}{3|K| \beta}+\mathrm{O}\left(T^{2}\right), & T \rightarrow 0, \\
\frac{c(0, T)}{k_{\mathrm{B}}}=\frac{\pi^{2}}{3|K| \beta}+\mathrm{O}\left(T^{2}\right), & T \rightarrow 0 .
\end{array}
$$

Hence both functions have a nonvanishing first derivative at $T=0$. As is well known, the entropy $s_{\Delta}(T)$ and the specific heat $c_{\Delta}(T)$ of a two-level system with energy gap $2 \Delta>0$ satisfy

$$
\begin{aligned}
\frac{s_{\Delta}(T)}{k_{\mathrm{B}}}=\log (2 \cosh (\beta \Delta))-\beta \Delta \tanh (\beta \Delta) & \sim 2 \beta \Delta \mathrm{e}^{-2 \beta \Delta}, & T & \rightarrow 0, \\
\frac{c_{\Delta}(T)}{k_{\mathrm{B}}}=(\beta \Delta)^{2} \operatorname{sech}^{2}(\beta \Delta) & \sim 4 \beta^{2} \Delta^{2} \mathrm{e}^{-2 \beta \Delta}, & T & \rightarrow 0,
\end{aligned}
$$

so that the derivatives of all orders of $s_{\Delta}(T)$ and $c_{\Delta}(T)$ vanish at $T=0$.

We shall next show that the previous analysis essentially holds for the HS and FI chains as well. To begin with, note that from Eqs. (5.1)-(5.2) it follows that

$$
f(0,0)=u(0,0)=\frac{K \varepsilon_{0}}{40}(1-\operatorname{sgn} K) \equiv u_{0},
$$


and thus

$$
\begin{aligned}
& f(0, T)-u_{0}=-\frac{1}{\beta} \int_{0}^{1} \log \left(1+\mathrm{e}^{-\frac{|K| \beta}{2} \varepsilon(x)}\right) \mathrm{d} x, \\
& u(0, T)-u_{0}=\frac{|K|}{2} \int_{0}^{1} \frac{\varepsilon(x)}{1+\mathrm{e}^{\frac{|K| \beta}{2} \varepsilon(x)}} \mathrm{d} x .
\end{aligned}
$$

On the other hand, the free energy $f_{\Delta}$ and the internal energy $u_{\Delta}$ of a two-level system with gap $2 \Delta$, when normalized so that $u_{\Delta}(0)=f_{\Delta}(0)=0$, are given by

$$
f_{\Delta}(T)=-\frac{1}{\beta} \log \left(1+\mathrm{e}^{-2 \beta \Delta}\right), \quad u_{\Delta}(T)=\frac{2 \Delta}{1+\mathrm{e}^{2 \beta \Delta}} .
$$

From the latter equation and Eqs. (5.15)- 5.16 , it follows that the thermodynamic functions of a two-level system with gap $2 \Delta$ are obtained by taking $\varepsilon(x)$ in Eqs. (5.3)-(5.4) and (5.18)-(5.19) as the constant

$$
\varepsilon_{\Delta}=\frac{4 \Delta}{|K|} .
$$

This observation suggests that, if the ratio $\Delta /|K|$ is chosen appropriately, the thermodynamic functions of a two-level system with gap $2 \Delta$ and those of a spin chain of HS type with coupling $K$ (and zero magnetic field) should behave in a qualitatively similar fashion. In order to establish the relation between $\Delta$ and $K$, we note that in the high-temperature limit one has

$$
\lim _{T \rightarrow \infty}\left[u(0, T)-u_{0}\right]=\frac{|K| \varepsilon_{0}}{4}, \quad \lim _{T \rightarrow \infty} u_{\Delta}(T)=\Delta .
$$

For both limits to coincide, we must have

$$
\frac{\Delta}{|K|}=\frac{\varepsilon_{0}}{4},
$$

i.e, $\varepsilon_{\Delta}$ should be equal to the average of $\varepsilon(x)$ over the interval $[0,1]$. In particular, in the case of the PF chain we have $\varepsilon_{0}=1 / 2$ (cf. Eq. (5.8)), which explains the choice $\Delta=|K| / 8$ in Fig. 1 . As an example, we have plotted in Fig. 2 (left) the specific heat per site of the PF, HS, and FI chains (with $\gamma=0,1$ in the latter case) compared to its counterpart for a two-level system with half-gap $\Delta=|K| / 8$. Here $K$ denotes the coupling of the PF chain and, following the previous analysis, we have fixed the corresponding couplings $K_{i}$ of the other chains by the requirement $K_{i} \int_{0}^{1} \varepsilon_{i}(x) \mathrm{d} x=K \int_{0}^{1} x \mathrm{~d} x=K / 2$.

We shall next derive the low temperature behavior of the thermodynamic functions of the HS and FI chains in the absence of a magnetic field. Our strategy shall be to obtain an asymptotic formula for the specific heat (5.4), which we shall then integrate to obtain similar formulas for the remaining thermodynamic functions.

Consider first the HS chain. Since in this case $\varepsilon(x)=x(1-x)$ is symmetric about $x=1 / 2$, we can write Eq. (5.4) as

$$
\frac{c(0, T)}{k_{\mathrm{B}}}=2 \lambda^{2} \int_{0}^{1 / 2} \frac{\varepsilon^{2}(x) \mathrm{e}^{-\lambda \varepsilon(x)}}{\left(1+\mathrm{e}^{-\lambda \varepsilon(x)}\right)^{2}} \mathrm{~d} x,
$$


where $\lambda \equiv|K| \beta / 2$ is a large parameter. Performing the change of variables $t=\lambda \varepsilon(x)$ we immediately obtain

$$
\frac{c(0, T)}{k_{\mathrm{B}}}=\frac{2}{\lambda} \int_{0}^{\lambda / 4} \frac{t^{2} \mathrm{e}^{-t}}{\left(1+\mathrm{e}^{-t}\right)^{2}} \frac{\mathrm{d} t}{\sqrt{1-\frac{4 t}{\lambda}}}=\frac{2}{\lambda} \int_{0}^{\infty} \frac{t^{2} \mathrm{e}^{-t}}{\left(1+\mathrm{e}^{-t}\right)^{2}} \mathrm{~d} t+\mathrm{O}\left(\lambda^{-2}\right)
$$

(see Appendix A for the details). The last integral is easily computed:

$$
\int_{0}^{\infty} \frac{t^{2} \mathrm{e}^{-t}}{\left(1+\mathrm{e}^{-t}\right)^{2}}=\sum_{n=1}^{\infty}(-1)^{n+1} n \int_{0}^{\infty} t^{2} \mathrm{e}^{-n t} \mathrm{~d} t=2 \sum_{n=1}^{\infty} \frac{(-1)^{n+1}}{n^{2}}=-2 \operatorname{Li}_{2}(-1)=\frac{\pi^{2}}{6}
$$

(cf. Eq. 4.27)), which combined with the previous equation yields

$$
\frac{c(0, T)}{k_{\mathrm{B}}}=\frac{2 \pi^{2}}{3|K| \beta}+O\left(T^{2}\right) .
$$

Note that the leading term in this formula is twice that in Eq. (5.14) for the PF chain, due to the fact that in the present case the leading contribution to the integral 5.4 comes from both endpoints. Apart from this inessential difference, the specific heat at low temperature behaves as its counterpart for the PF chain, i.e., it increases linearly with the temperature.

Consider next the FI chain. If $\gamma \neq 0$, proceeding as before we obtain

$$
\begin{aligned}
\frac{c(0, T)}{k_{\mathrm{B}}} & =\lambda^{2} \int_{0}^{1} \frac{\varepsilon^{2}(x) \mathrm{e}^{-\lambda \varepsilon(x)}}{\left(1+\mathrm{e}^{-\lambda \varepsilon(x)}\right)^{2}} \mathrm{~d} x=\frac{1}{\lambda} \int_{0}^{(\gamma+1) \lambda} \frac{t^{2} \mathrm{e}^{-t}}{\left(1+\mathrm{e}^{-t}\right)^{2}} \frac{\mathrm{d} t}{\sqrt{\gamma^{2}+\frac{4 t}{\lambda}}} \\
& =\frac{1}{\gamma \lambda} \int_{0}^{\infty} \frac{t^{2} \mathrm{e}^{-t}}{\left(1+\mathrm{e}^{-t}\right)^{2}} \mathrm{~d} t+\mathrm{O}\left(\lambda^{-2}\right)=\frac{\pi^{2}}{3 \gamma|K| \beta}+O\left(T^{2}\right) .
\end{aligned}
$$

Again, in the low temperature range the specific heat increases linearly with the temperature.

Finally, for the FI chain with $\gamma=0$ we have

$$
\begin{aligned}
& \frac{c(0, T)}{k_{\mathrm{B}}}=\lambda^{2} \int_{0}^{1} \frac{x^{4} \mathrm{e}^{-\lambda x^{2}}}{\left(1+\mathrm{e}^{-\lambda x^{2}}\right)^{2}} \mathrm{~d} x=\frac{1}{2 \sqrt{\lambda}} \int_{0}^{\lambda} \frac{t^{3 / 2} \mathrm{e}^{-t}}{\left(1+\mathrm{e}^{-t}\right)^{2}} \mathrm{~d} t \\
& =\frac{1}{2 \sqrt{\lambda}} \int_{0}^{\infty} \frac{t^{3 / 2} \mathrm{e}^{-t}}{\left(1+\mathrm{e}^{-t}\right)^{2}} \mathrm{~d} t+\mathrm{O}\left(\lambda \mathrm{e}^{-\lambda}\right),
\end{aligned}
$$

where the last integral can again be exactly evaluated:

$$
\begin{aligned}
\int_{0}^{\infty} \frac{t^{3 / 2} \mathrm{e}^{-t}}{\left(1+\mathrm{e}^{-t}\right)^{2}} \mathrm{~d} t & =\sum_{n=1}^{\infty}(-1)^{n+1} n \int_{0}^{\infty} t^{3 / 2} \mathrm{e}^{-n t} \mathrm{~d} t \\
& =\sum_{n=1}^{\infty} \frac{(-1)^{n+1}}{n^{3 / 2}} \int_{0}^{\infty} t^{3 / 2} \mathrm{e}^{-t} \mathrm{~d} t=\eta(3 / 2) \Gamma(5 / 2)=\frac{3 \sqrt{\pi}}{4} \eta(3 / 2),
\end{aligned}
$$

where

$$
\eta(z)=\sum_{n=1}^{\infty} \frac{(-1)^{n+1}}{n^{z}}
$$


is Dirichlet's eta function. Using the elementary relation

$$
\eta(z)=\left(1-2^{1-z}\right) \zeta(z)
$$

where $\zeta(z)$ is Riemann's zeta function, we finally obtain

$$
\int_{0}^{\infty} \frac{t^{3 / 2} \mathrm{e}^{-t}}{\left(1+\mathrm{e}^{-t}\right)^{2}} \mathrm{~d} t=\frac{3 \sqrt{\pi}}{4}\left(1-\frac{1}{\sqrt{2}}\right) \zeta(3 / 2),
$$

and hence

$$
\frac{c(0, T)}{k_{\mathrm{B}}}=\frac{3}{8}(\sqrt{2}-1) \zeta(3 / 2) \sqrt{\frac{\pi}{|K| \beta}}+\mathrm{O}\left(\beta \mathrm{e}^{-|K| \beta / 2}\right) .
$$

In contrast with the previous cases, the specific heat $c(0, T)$ has now an infinite first derivative at $T=0$; see Fig. 2(right). Hence the FI chain with $\gamma=B=0$ exhibits a second-order phase transition at zero temperature.

Since asymptotic expansions can in general be integrated termwise to yield valid asymptotic expansions [47], integrating the asymptotic formulas (5.14) and (5.22)-(5.24) we can readily derive the low temperature behavior of the remaining thermodynamic functions of all spin chains of HS type. Thus, for the FI chain with $\gamma=0$ we have

$$
\begin{aligned}
& f(0, T)=u_{0}-\frac{4 \eta}{3} \beta^{-3 / 2}+\mathrm{O}\left(\beta^{-2} \mathrm{e}^{-|K| \beta / 2}\right) \\
& u(0, T)=u_{0}+\frac{2 \eta}{3} \beta^{-3 / 2}+\mathrm{O}\left(\beta^{-1} \mathrm{e}^{-|K| \beta / 2}\right) \\
& \frac{s(0, T)}{k_{\mathrm{B}}}=2 \eta \beta^{-1 / 2}+\mathrm{O}\left(\mathrm{e}^{-|K| \beta / 2}\right)
\end{aligned}
$$

where we have set

$$
\eta=\frac{3}{8}(\sqrt{2}-1) \zeta(3 / 2) \sqrt{\frac{\pi}{|K|}} \simeq 0.719227|K|^{-1 / 2} .
$$

For all other chains of HS type, the analogous results can be concisely summarized as follows:

$$
\begin{aligned}
& f(0, T)=u_{0}-\frac{\eta \pi^{2}}{6|K| \beta^{2}}+\mathrm{O}\left(T^{3}\right) \\
& u(0, T)=u_{0}+\frac{\eta \pi^{2}}{6|K| \beta^{2}}+\mathrm{O}\left(T^{3}\right) \\
& \frac{s(0, T)}{k_{\mathrm{B}}}=\frac{\eta \pi^{2}}{3|K| \beta}+\mathrm{O}\left(T^{2}\right),
\end{aligned}
$$

where now

$$
\eta= \begin{cases}2, & \text { for the HS chain } \\ 1, & \text { for the PF chain } \\ 1 / \gamma, & \text { for the FI chainwith } \gamma \neq 0\end{cases}
$$



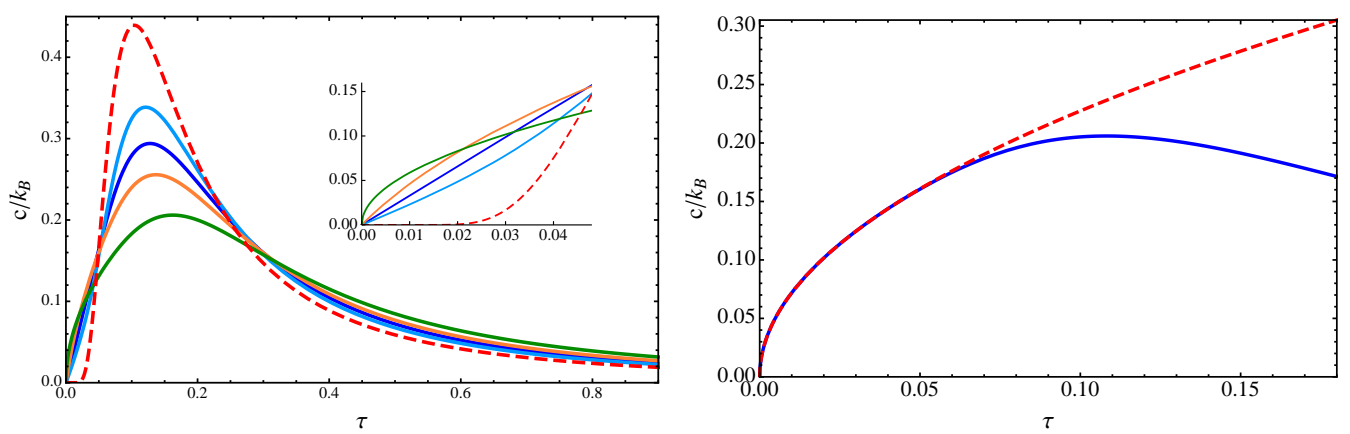

Figure 2: Left: specific heat per site of the PF (blue), HS (light blue), and FI ( $\gamma=1$, orange; $\gamma=0$, green) chains with zero magnetic field and of a two-level system with half-gap $\Delta=|K| / 8$ (dashed red line) as functions of the dimensionless temperature $\tau=(|K| \beta)^{-1}$. Right: specific heat per site of the FI chain with $\gamma=0$ (solid blue line) versus its lowtemperature approximation 5.24 (dashed red line).

\section{The zero temperature limit}

In this section we shall analyze the low temperature behavior of the thermodynamic functions of a spin chain of HS type in the presence of a nonzero magnetic field. In the ferromagnetic case $(K>0)$, we already know from the previous section that there must be a phase transition at $T=B=0$. This fact is also apparent from Eq. (4.19) for the magnetization, which for $K>0$ implies that

$$
\lim _{T \rightarrow 0} \mu(B, T)=\operatorname{sgn} B .
$$

The latter result can be corroborated by a computation of the zero-temperature magnetization per site directly from its definition. Indeed, from Eqs. 4.2. 4.3 it follows that

$$
\mu(B, 0)=\lim _{N \rightarrow \infty} \frac{1}{N}\left\langle\sum_{i=1}^{N} \sigma_{i}\right\rangle_{\mathrm{g}},
$$

where $\langle\cdot\rangle_{\mathrm{g}}$ denotes the average over all ground states. When $B \neq 0$ and $K>0$ the ground state is obtained by taking $\sigma_{i}=\operatorname{sgn} B$ for all $i$ in Eq. (3.10), and is therefore nondegenerate. Hence for $B \neq 0$ we have

$$
\mu(B, 0)=\lim _{N \rightarrow \infty} \frac{1}{N} \cdot N \operatorname{sgn} B=\operatorname{sgn} B,
$$

as before. On the other hand, when $B=0$ the ground states are obtained from sequences of the form $\sigma=(1, \ldots, 1,-1, \ldots,-1)$. The average of $\sum_{i=1}^{N} \sigma_{i}$ over such sequences clearly vanishes, since the contributions of the sequences with $k 1$ 's and $N-k 1$ 's cancel each other. Thus $\mu(0,0)=$ 0 , again in agreement with Eq. (6.1).

In the antiferromagnetic case, it can be shown from Eq. (4.19) that there is a phase transition at zero temperature and magnetic field $B= \pm B_{\mathrm{s}}$, where the saturation field $B_{\mathrm{s}}$ is given by

$$
B_{\mathrm{S}}=\frac{1}{2}|K| \max _{x \in[0,1]} \varepsilon(x)= \begin{cases}\frac{|K|}{8}, & \text { for the HS chain } \\ \frac{|K|}{2}, & \text { for the PF chain } \\ \frac{|K|}{2}(\gamma+1), & \text { for the FI chain. }\end{cases}
$$


Indeed, when $K<0$ we can rewrite Eq. (4.19) as

$$
|\mu(B, T)|=\kappa \int_{0}^{1 / \kappa}\left[1+4\left(1-\mathrm{e}^{-2 \beta|B|}\right)^{-2} \mathrm{e}^{\lambda \varphi(x)}\right]^{-1 / 2} \mathrm{~d} x,
$$

where $\lambda=|K| \beta \rightarrow \infty$,

$$
\kappa= \begin{cases}1, & \text { for the PF and FI chains } \\ 2, & \text { for the HS chain, }\end{cases}
$$

and

$$
\varphi(x)=\varepsilon(x)-\frac{2|B|}{|K|} \equiv \varepsilon(x)-b .
$$

Note that the value $\kappa=2$ for the HS chain is due to the symmetry of $\varepsilon(x)=x(1-x)$ about $x=1 / 2$ in this case. If $|B|>B_{\mathrm{S}}$ then $b>\max _{x \in[0,1]} \varepsilon(x)=\max _{x \in[0, k]} \varepsilon(x)$, so that for all $x \in[0, \kappa]$ we have $\varphi(x)<0$ and $\mathrm{e}^{\lambda \varphi(x)} \rightarrow 0$ as $\lambda \rightarrow \infty$. Hence

$$
|B|>B_{\mathrm{s}} \Longrightarrow \lim _{T \rightarrow 0}|\mu(B, T)|=\kappa \int_{0}^{1 / \kappa} \mathrm{d} x=1,
$$

i.e, for $|B|>B_{\mathrm{s}}$ the magnetization per site saturates. On the other hand, if $|B| \leqslant B_{\mathrm{s}}$ we have $\varphi(x)<0$ for $0 \leqslant x<x_{0}$ and $\varphi(x)>0$ for $x_{0}<x \leqslant \kappa$, where $x_{0}$ denotes the unique root in $[0, \kappa]$ of the equation $\varphi(x)=0$, i.e,

$$
\varepsilon\left(x_{0}\right)=b, \quad \text { with } \quad x_{0} \in[0, \kappa] .
$$

Thus in this case we have

$$
\lim _{\lambda \rightarrow \infty} \mathrm{e}^{\lambda \varphi(x)}= \begin{cases}0, & \text { for } 0 \leqslant x<x_{0} \\ \infty, & \text { for } x_{0}<x \leqslant \kappa,\end{cases}
$$

and therefore

$$
|B| \leqslant B_{\mathrm{s}} \quad \Longrightarrow \quad \lim _{T \rightarrow 0}|\mu(B, T)|=\kappa \int_{0}^{x_{0}} \mathrm{~d} x=\kappa x_{0} .
$$

Note that the RHS in both Eqs. (6.6) and (6.8) tends to 1 as $|B| \rightarrow B_{\mathrm{s}}$, since clearly $x_{0}$ tends to $1 / \kappa$ as $|B| \rightarrow B_{\mathrm{s}}$.

For the HS chain we have $\varepsilon(x)=x(1-x)$, and hence

$$
x_{0}=\frac{1}{2}-\sqrt{\frac{1}{4}-b} \equiv \frac{1}{2}-\sqrt{\frac{1}{4}-\frac{2|B|}{|K|}} .
$$

Equations (6.3) and 6.6)-(6.8) (with $\kappa=2)$ immediately yield

$$
\lim _{T \rightarrow 0} \mu(B, T)= \begin{cases}\operatorname{sgn} B\left(1-\sqrt{1-\frac{8|B|}{|K|}}\right), & |B| \leqslant \frac{|K|}{8} \\ \operatorname{sgn} B, & |B|>\frac{|K|}{8},\end{cases}
$$


which was first derived by Haldane using the spinon gas formalism [23]. Likewise, for the PF chain we have $\kappa=1, \varepsilon(x)=x$ and thus $x_{0}=b \equiv 2|B| /|K|$, so that

$$
\lim _{T \rightarrow 0} \mu(B, T)= \begin{cases}\frac{2 B}{|K|}, & |B| \leqslant \frac{|K|}{2} \\ \operatorname{sgn} B, & |B|>\frac{|K|}{2}\end{cases}
$$

Finally, in the case of the FI chain $\kappa=1, \varepsilon(x)=x(\gamma+x)$,

$$
x_{0}=-\frac{\gamma}{2}+\sqrt{\frac{\gamma^{2}}{4}+b} \equiv-\frac{\gamma}{2}+\sqrt{\frac{\gamma^{2}}{4}+\frac{2|B|}{|K|}}
$$

and thus

$$
\lim _{T \rightarrow 0} \mu(B, T)= \begin{cases}\frac{1}{2} \operatorname{sgn} B\left(\sqrt{\gamma^{2}+\frac{8|B|}{|K|}}-\gamma\right), & |B| \leqslant \frac{|K|}{2}(\gamma+1) \\ \operatorname{sgn} B, & |B|>\frac{|K|}{2}(\gamma+1),\end{cases}
$$

in agreement with the result in Ref. [6].

From Eqs. (6.10)- 6.13) it is apparent that, although the magnetization per site is continuous at $B= \pm B_{\mathrm{s}}$, the susceptibility $\chi=\frac{\partial \mu}{\partial B}$ has a discontinuity at these points (cf. Fig. 3 ). Hence, in the antiferromagnetic case all three chains of HS type present a second-order phase transition at $T=0$ and $B= \pm B_{\mathrm{s}}$, where the saturation field $B_{\mathrm{s}}$ is explicitly given in Eq. 6.3). The precise behavior of the zero-temperature magnetization and susceptibility for $|B| \leqslant B_{\mathrm{s}}$ is, however, quite different for each type of chain. Indeed, in the case of the HS chain the susceptibility is an increasing function of $B$ for $0 \leqslant B<B_{\mathrm{s}}$, and diverges as $\left(B_{\mathrm{s}}-|B|\right)^{-1 / 2}$ when $B \rightarrow \pm B_{\mathrm{s}} \mp$. On the other hand, for both the PF and FI chains the susceptibility has only a jump discontinuity at the critical points $B= \pm B_{\mathrm{s}}$. When $0 \leqslant B<B_{\mathrm{s}}$ the susceptibility is constant for the PF chain, while for the FI chain it decreases monotonically. Finally, it should be noted that the first derivative of the zero-temperature susceptibility has a jump discontinuity at $B=0$ for the FI chain with $\gamma>0$ and the HS chain, while it diverges as $|B|^{-1 / 2}$ at this point for the FI chain with $\gamma=0$ (cf. Fig. 3 ).

As in the ferromagnetic case, the zero-temperature magnetization can also be computed directly taking advantage of the exact knowledge of the spectrum. Indeed, consider first the PF and FI chains. In this case the term proportional to $K=-|K|$ in Eqs. (4.2)-(4.3) is minimized by the sequence $\sigma_{0}=(\ldots,-1,1, \ldots,-1,1,-1,1)$, characteristic of an antiferromagnetic system, while the term proportional to $B$ is again minimized by the sequence with all components equal to $\operatorname{sgn} B$. It is therefore clear that the ground state is obtained by flipping the components of $\sigma_{0}$ equal to $-\operatorname{sgn} B$ starting from the left, until the resulting decrease $2|B|$ in the term proportional to $B$ in Eq. 4.2) is offset by the increase $|K| \varepsilon_{i} \equiv|K| \varepsilon\left(x_{i}\right)$ in the other term. Thus if $|B|>\frac{1}{2}|K| \max _{1 \leqslant i \leqslant N} \varepsilon\left(x_{i}\right)=B_{\mathrm{s}}$ the ground state is again obtained by taking $\sigma_{i}=\operatorname{sgn} B$ for all $i=1, \ldots, N$, and the magnetization attains its saturation value $\operatorname{sgn} B$. On the other hand, when $|B| \leqslant B_{\mathrm{s}}$ the ground state is obtained from the sequence

$$
\overbrace{(\operatorname{sgn} B, \ldots, \operatorname{sgn} B}^{i},-1,1,-1,1, \ldots,-1,1)
$$

where for large $N$ the index $i$ is (approximately) determined by the condition

$$
|K| \varepsilon\left(x_{i}\right) \simeq 2|B| .
$$



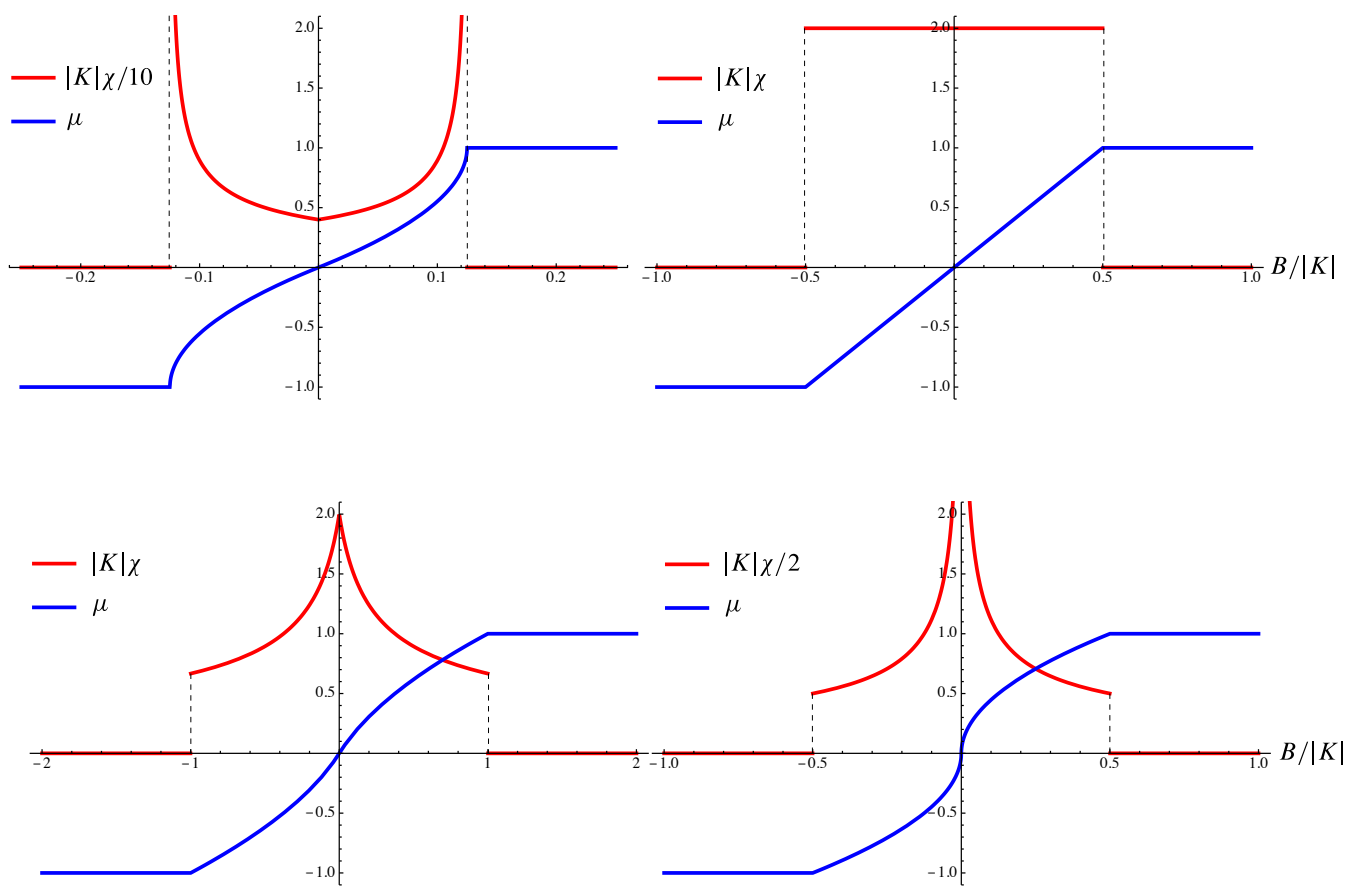

Figure 3: Top to bottom and left to right: zero-temperature magnetization and susceptibility per site of the HS, PF, and FI chains (with $\gamma=1$ and $\gamma=0$ for the latter chain) as functions of the external magnetic field $B$.

By Eq. 6.2), the magnetization per site is therefore given ${ }^{5}$

$$
\mu(B, 0)=\operatorname{sgn} B \cdot \lim _{N \rightarrow \infty} \frac{i}{N} \equiv \operatorname{sgn} B \cdot \lim _{N \rightarrow \infty} x_{i} .
$$

Taking into account that $\lim _{N \rightarrow \infty} x_{i}=x_{0}$, where as before $x_{0}$ is determined by Eq. 6.7) (with $\kappa=1$ ), we obtain the following formula for the zero-temperature magnetization per site for $|B| \leqslant B_{\mathrm{s}}$ :

$$
\mu(B, 0)=\operatorname{sgn} B \cdot x_{0} \text {. }
$$

The latter equation clearly agrees with Eq. 6.8) with $\kappa=1$. For the HS chain the analysis is very similar, except that the sequence $\sigma_{0}$ minimizing the term proportional to $K$ in Eq. 4.2) is now of the form

$$
\sigma_{0}=(\ldots,-1,1, \ldots,-1,1,-\underset{\substack{\downarrow \\[N / 2]}}{1}, 1,-1,1 \ldots,-1,1, \ldots) .
$$

This is due to the fact that in this case the dispersion relation $\varepsilon(x)$ has a maximum at $x=1 / 2$, is symmetric about this point, and is monotonically increasing in the interval $[0,1 / 2]$. For the same

\footnotetext{
${ }^{5}$ More rigorously, for large $N$ there could be a small number of ground states with corresponding sequences almost identical to 6.14, so that the average of $\sum_{i=1}^{N} \sigma_{i}$ over these ground states is approximately equal to its value on the latter state.
} 
reason, if $|B|<B_{\mathrm{s}}$ the ground state is now obtained from a sequence of the form ${ }^{6}$

$$
\overbrace{(\operatorname{sgn} B, \ldots, \operatorname{sgn} B}^{i},-1,1, \ldots,-1,1, \overbrace{\operatorname{sgn} B, \ldots, \operatorname{sgn} B}^{i}),
$$

where $i$ is again approximately determined by Eq. (6.15). Thus in this case Eq. 6.16 should be replaced by

$$
\mu(B, 0)=\operatorname{sgn} B \lim _{N \rightarrow \infty} \frac{2 i}{N}=2 \operatorname{sgn} B \lim _{N \rightarrow \infty} x_{i}=2 \operatorname{sgn} B \cdot x_{0},
$$

which is essentially Eq. 6.8 for $\kappa=2$.

We shall next analyze the low-temperature behavior of the thermodynamic functions of the antiferromagnetic chains in the physically more interesting regime $0<|B|<B_{\mathrm{s}}$. To this end, we start by rewriting Eq. (4.18) for the free energy per site as

$$
-\beta(f(B, T)+|B|)=\int_{0}^{1} \log \left[\frac{1+\mathrm{e}^{-2 \beta|B|}}{2}+\frac{1}{2} \sqrt{\left(1-\mathrm{e}^{-2 \beta|B|}\right)^{2}+4 \mathrm{e}^{\lambda \varphi(x)}}\right] \mathrm{d} x,
$$

where $\lambda \equiv|K| \beta \rightarrow \infty$ and $\varphi(x)$ is given by Eq. 6.5. It is straightforward to show that the function

$$
g(x, z)=\log \left[\frac{1+z}{2}+\frac{1}{2} \sqrt{(1-z)^{2}+4 \mathrm{e}^{\lambda \varphi(x)}}\right]
$$

satisfies

$$
0 \leqslant \frac{\partial g(x, z)}{\partial z} \leqslant \frac{1}{2} \mathrm{e}^{-\frac{\lambda}{2} \varphi(x)}, \quad z \geqslant 0 .
$$

Integrating this inequality with respect to $z$ over the interval $\left[0, \mathrm{e}^{-2 \beta|B|}\right]$ we obtain

$$
0 \leqslant g\left(x, \mathrm{e}^{-2 \beta|B|}\right)-g(x, 0) \leqslant \frac{1}{2} \mathrm{e}^{-\frac{1}{2} \lambda \varphi(x)} \mathrm{e}^{-2 \beta|B|}=\frac{1}{2} \mathrm{e}^{-\frac{\lambda}{2} \varepsilon(x)} \mathrm{e}^{-\beta|B|} \leqslant \frac{1}{2} \mathrm{e}^{-\beta|B|} .
$$

Integrating now with respect to $x$ over $[0,1]$ and taking into account Eqs. 6.18)- 6.19] we easily arrive at the asymptotic relation

$$
\begin{aligned}
-\beta(f(B, T)+|B|) & =\int_{0}^{1} \log \left(\frac{1+\sqrt{1+4 \mathrm{e}^{\lambda \varphi(x)}}}{2}\right) \mathrm{d} x+\mathrm{O}\left(\mathrm{e}^{-\beta|B|}\right) \\
& =\kappa \int_{0}^{1 / \kappa} \log \left(\frac{1+\sqrt{1+4 \mathrm{e}^{\lambda \varphi(x)}}}{2}\right) \mathrm{d} x+\mathrm{O}\left(\mathrm{e}^{-\beta|B|}\right),
\end{aligned}
$$

where we have used the notation introduced in Eq. 6.4). Since $\varphi(x)$ is negative for $0 \leqslant x<x_{0}$ and positive for $x_{0}<x \leqslant \kappa$, it is convenient to rewrite the latter equation as

$$
\begin{aligned}
-\frac{\beta}{\kappa}(f(B, T)+|B| & \left.+\frac{\kappa|K|}{2} \int_{x_{0}}^{1 / \kappa} \varphi(x) \mathrm{d} x\right)=\int_{0}^{x_{0}} \log \left(\frac{1+\sqrt{1+4 \mathrm{e}^{\lambda \varphi(x)}}}{2}\right) \mathrm{d} x \\
& +\int_{x_{0}}^{1 / \kappa} \log \left(\frac{1}{2} \mathrm{e}^{-\frac{\lambda}{2} \varphi(x)}+\sqrt{1+\frac{1}{4} \mathrm{e}^{-\lambda \varphi(x)}}\right) \mathrm{d} x+\mathrm{O}\left(\mathrm{e}^{-\beta|B|}\right) .
\end{aligned}
$$

${ }^{6}$ As before, in general there could be a small number of ground states corresponding to sequences nearly equal to Eq. 6.17), but this does not affect the conclusion for the reason given in the previous footnote 
Consider now the first integral in the RHS of the previous equation. Performing the change of variable $t=-\lambda \varphi(x)$ and proceeding as in the previous section we readily obtain

$$
\begin{array}{r}
\int_{0}^{x_{0}} \log \left(\frac{1+\sqrt{1+4 \mathrm{e}^{\lambda \varphi(x)}}}{2}\right) \mathrm{d} x=\frac{1}{\lambda} \int_{0}^{\lambda b} \log \left(\frac{1+\sqrt{1+4 \mathrm{e}^{-t}}}{2}\right) \frac{\mathrm{d} t}{\varepsilon^{\prime}(x(t))} \\
=\frac{1}{\lambda \varepsilon^{\prime}\left(x_{0}\right)} \int_{0}^{\infty} \log \left(\frac{1+\sqrt{1+4 \mathrm{e}^{-t}}}{2}\right) \mathrm{d} t+\mathrm{O}\left(\lambda^{-2}\right),
\end{array}
$$

where the error term has been estimated as in Appendix A. Note that if $0<|B|<B_{\mathrm{s}}$ we must have $0<x_{0}<\kappa$, and consequently $\varepsilon^{\prime}\left(x_{0}\right)>0$. Observe also that, in order for the above estimate to be valid, we must have $\lambda b \gg 1$, or (disregarding an inessential factor of 2)

$$
\beta|B| \gg 1 \text {. }
$$

The second integral in Eq. 6.21) can be similarly dealt with by the change of variable $t=\lambda \varphi(x)$ :

$$
\begin{aligned}
\int_{x_{0}}^{1 / \kappa} \log ( & \left(\frac{1}{2} \mathrm{e}^{-\frac{\lambda}{2} \varphi(x)}+\sqrt{1+\frac{1}{4} \mathrm{e}^{-\lambda \varphi(x)}}\right) \mathrm{d} x \\
= & \frac{1}{\lambda} \int_{0}^{\lambda \varphi(1 / \kappa)} \log \left(\frac{1}{2} \mathrm{e}^{-t / 2}+\sqrt{1+\frac{1}{4} \mathrm{e}^{-t}}\right) \frac{\mathrm{d} t}{\varepsilon^{\prime}(x(t))} \\
= & \frac{1}{\lambda \varepsilon^{\prime}\left(x_{0}\right)} \int_{0}^{\infty} \log \left(\frac{1}{2} \mathrm{e}^{-t / 2}+\sqrt{1+\frac{1}{4} \mathrm{e}^{-t}}\right) \mathrm{d} t+\mathrm{O}\left(\lambda^{-2}\right) .
\end{aligned}
$$

This formula is correct provided that $\lambda \varphi(1 / \kappa)=\beta(|K| \varepsilon(1 / \kappa)-2|B|) \gg 1$, or equivalently (since $\left.|K| \varepsilon(1 / \kappa)=2 B_{\mathrm{s}}\right)$,

$$
\beta\left(B_{\mathrm{s}}-|B|\right) \gg 1 .
$$

Combining Eq. 6.21) with Eqs. (6.22)- 6.23 we obtain

$$
-\frac{\beta}{\kappa}\left(f(B, T)+|B|+\frac{\kappa|K|}{2} \int_{x_{0}}^{1 / \kappa} \varphi(x) \mathrm{d} x\right)=\frac{I_{1}+I_{2}}{\lambda \varepsilon^{\prime}\left(x_{0}\right)}+\mathrm{O}\left(\lambda^{-2}\right),
$$

where

$$
I_{1}=\int_{0}^{\infty} \log \left(\frac{1+\sqrt{1+4 \mathrm{e}^{-t}}}{2}\right) \mathrm{d} t, \quad I_{2}=\int_{0}^{\infty} \log \left(\frac{1}{2} \mathrm{e}^{-t / 2}+\sqrt{1+\frac{1}{4} \mathrm{e}^{-t}}\right) \mathrm{d} t
$$

and we have omitted an exponentially small term $\mathrm{O}\left(\mathrm{e}^{-\beta|B|}\right) \ll \mathrm{O}\left(\lambda^{-2}\right)$. Using the value of these integrals in Eqs. (B.2)-B.4) of Appendix B we finally obtain the asymptotic formula

$$
f(B, T)=-|B|-\frac{\kappa|K|}{2} \int_{x_{0}}^{1 / \kappa} \varphi(x) \mathrm{d} x-\frac{\kappa \pi^{2}}{6|K| \beta^{2} \varepsilon^{\prime}\left(x_{0}\right)}+\mathrm{O}\left(T^{3}\right),
$$

provided that

$$
\frac{1}{\beta} \ll|B| \ll B_{\mathrm{s}}-\frac{1}{\beta} .
$$

Since Eq. 6.26 is an asymptotic power series expansion in $\beta$, it may be differentiated termwise [47] to yield corresponding expansions for the remaining thermodynamic quantities 
as $T \rightarrow 0$. In the first place, taking into account that $x_{0}$ and $\varphi(x)$ are independent of the temperature we immediately obtain

$$
\begin{aligned}
u(B, T) & =-|B|-\frac{\kappa|K|}{2} \int_{x_{0}}^{1 / \kappa} \varphi(x) \mathrm{d} x+\frac{\kappa \pi^{2}}{6|K| \beta^{2} \varepsilon^{\prime}\left(x_{0}\right)}+\mathrm{O}\left(T^{3}\right), \\
\frac{c(B, T)}{k_{\mathrm{B}}} & =\frac{\kappa \pi^{2}}{3|K| \beta \varepsilon^{\prime}\left(x_{0}\right)}+\mathrm{O}\left(T^{2}\right), \\
\frac{s(B, T)}{k_{\mathrm{B}}} & =\frac{\kappa \pi^{2}}{3|K| \beta \varepsilon^{\prime}\left(x_{0}\right)}+\mathrm{O}\left(T^{2}\right) .
\end{aligned}
$$

In particular, in the case of the HS chain Eq. 6.30) is in agreement with Haldane's original result [23].

In order to compute the asymptotic expansion of the magnetization, we observe that, by Eq. (6.7),

$$
\frac{\partial x_{0}}{\partial B}=\frac{2 \operatorname{sgn} B}{|K| \mathcal{E}^{\prime}\left(x_{0}\right)},
$$

and, since $\varphi\left(x_{0}\right)=0$,

$$
\frac{\partial}{\partial B} \int_{x_{0}}^{1 / \kappa} \varphi(x) \mathrm{d} x=\int_{x_{0}}^{1 / \kappa} \frac{\partial \varphi(x)}{\partial B} \mathrm{~d} x=\frac{2 \operatorname{sgn} B}{|K|}\left(x_{0}-\frac{1}{\kappa}\right) .
$$

We thus have

$$
|\mu(B, T)|=\kappa x_{0}-\frac{\kappa \pi^{2} \varepsilon^{\prime \prime}\left(x_{0}\right)}{3 K^{2} \beta^{2} \varepsilon^{\prime}\left(x_{0}\right)^{3}}+O\left(T^{3}\right) .
$$

Note, in particular, that the leading term in the latter expansion coincides with Eq. (6.8). Using the values of $x_{0}$ and $\kappa$ in Eqs. (6.9)-6.12) and (6.4) we easily obtain

$$
|\mu(B, T)|=1-\sqrt{1-\frac{8|B|}{|K|}}+\frac{4 \pi^{2}}{3 K^{2} \beta^{2}}\left(1-\frac{8|B|}{|K|}\right)^{-3 / 2}+\mathrm{O}\left(T^{3}\right),
$$

for the HS chain, and

$$
|\mu(B, T)|=\frac{1}{2} \sqrt{\gamma^{2}+\frac{8|B|}{|K|}}-\frac{\gamma}{2}-\frac{2 \pi^{2}}{3 K^{2} \beta^{2}}\left(\gamma^{2}+\frac{8|B|}{|K|}\right)^{-3 / 2}+\mathrm{O}\left(T^{3}\right),
$$

for the FI chain. In the case of the PF chain the $\mathrm{O}\left(T^{2}\right)$ term in Eq. 6.31) vanishes. In fact, in this case Eq. 4.30) more directly yields

$$
|\mu(B, T)|=\frac{2|B|}{|K|}-\frac{\mathrm{e}^{-\frac{\beta}{2}(|K|-2|B|)}}{|K| \beta}+\mathrm{O}\left(\frac{1}{\beta} \mathrm{e}^{-\beta \min \left(\frac{|K|}{2}+|B|,|K|-2|B|\right)}\right) .
$$

The approximations 6.32)- 6.34) are in excellent agreement with the exact result 4.19) (or (4.30), for the PF chain) provided that the magnetic field is in the range (6.27); see, e.g., Fig. 4 for the HS and FI chains. 

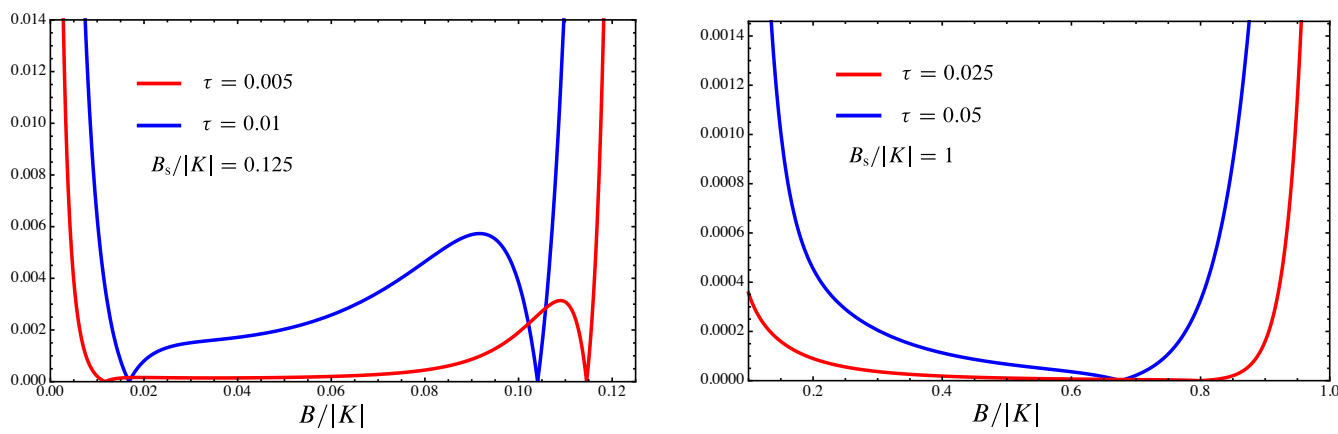

Figure 4: Left: absolute value of the relative error of the approximation 6.32 for HS the chain for different values of the dimensionless temperature. Right: similar plot for the approximation 6.33 for the FI chain with $\gamma=1$.

\section{Connection with the one-dimensional Ising model}

We have seen in Section 3 that the chains (1.6)-(1.7) with spin $1 / 2$ are isospectral to the classical inhomogeneous Ising model in an external (also inhomogeneous) magnetic field defined by Eqs. (3.11) and (3.12). In this section we shall exploit this fact to study the connection between the above chains and the standard (homogeneous) Ising model, whose Hamiltonian we shall conveniently take as

$$
\mathcal{H}_{J}=J \sum_{i=1}^{N}\left(1-S_{i} S_{i+1}\right)-B \sum_{i=1}^{N} S_{i}, \quad S_{N+1} \equiv S_{1} .
$$

With this normalization, the free energy per site in the thermodynamic limit is given by [48]

$$
f_{J}(T, B)=-\frac{1}{\beta} \log \left(\cosh (\beta B)+\sqrt{\sinh (\beta B)^{2}+\mathrm{e}^{-4 \beta J}}\right),
$$

which is clearly reminiscent of the analogous Eq. (4.18) for the spin chains of Haldane-Shastry type. In fact, we can give a heuristic derivation of the latter equation from Eqs. (3.11)-(3.12) and the previous formula for the free energy per site of the Ising model. To this end, note first of all that the latter equations can be written as

$$
E(\sigma)=\frac{K}{4} \sum_{i=1}^{N-1} \varepsilon_{i}\left(1-\sigma_{i} \sigma_{i+1}\right)-\sum_{i=1}^{N} \mathcal{B}(i) \sigma_{i}
$$

where

$$
\mathcal{B}(i) \equiv B+\frac{K}{4}\left(\varepsilon_{i}-\varepsilon_{i-1}\right) .
$$

We saw in Section 4 that when $N \gg 1$ the term $\varepsilon_{i}-\varepsilon_{i-1}$ is $\mathrm{O}\left(N^{-1}\right)$, so that in this limit we have

$$
E(\boldsymbol{\sigma}) \simeq \frac{K}{4} \sum_{i=1}^{N-1} \varepsilon_{i}\left(1-\sigma_{i} \sigma_{i+1}\right)-B \sum_{i=1}^{N} \sigma_{i}
$$


Proceeding as in the latter section, from the previous equation we can show that when $N \gg 1$ the partition function of the chains (1.6)-(1.7) is approximately given by

$$
\mathcal{Z} \simeq \prod_{i=1}^{N-1} \lambda_{+}\left(\frac{K \varepsilon_{i}}{4}\right),
$$

where $\lambda_{+}(J)$ denotes the largest eigenvalue of the transfer matrix the Ising model $(7.1)$ with coupling $J$. Hence, in the thermodynamic limit the free energy per site of the chains 1.6-1.7p is given by

$$
f(B, T)=-\lim _{N \rightarrow \infty} \frac{\log \mathcal{Z}}{N \beta}=-\lim _{N \rightarrow \infty} \frac{1}{N \beta} \sum_{i=1}^{N-1} \log \lambda_{+}\left(\frac{K \varepsilon_{i}}{4}\right) .
$$

On the other hand, when $N \rightarrow \infty$ the partition function $\mathcal{Z}_{J}$ of the Ising model is related to the largest eigenvalue $\lambda_{+}(J)$ of its transfer matrix by [48]

$$
\mathcal{Z}_{J} \simeq \lambda_{+}(J)^{N}
$$

so that

$$
f_{J}(B, T)=-\lim _{N \rightarrow \infty} \frac{\log \mathcal{Z}_{J}}{N \beta}=-\frac{1}{\beta} \log \lambda_{+}(J) .
$$

Inserting this identity (with $J=\frac{K \varepsilon_{i}}{4}$ ) into Eq. (7.3) we obtain

$$
f(B, T)=\lim _{N \rightarrow \infty} \frac{1}{N} \sum_{i=1}^{N-1} f_{\frac{K_{\varepsilon_{i}}}{4}}(B, T)=\int_{0}^{1} f_{\frac{K \varepsilon(x)}{4}}(B, T) \mathrm{d} x,
$$

where we have used the fact that $\varepsilon_{i}=\varepsilon\left(x_{i}\right) \equiv \varepsilon(i / N)$. Equation 4.18$)$ for the free energy of the spin chains (1.6)-(1.7) readily follows from the previous equation and the formula (7.2) for $f_{J}$.

Equation (7.4) for the free energy of the Haldane-Shastry spin chains admits a statistical interpretation that we shall discuss next. Indeed, let us first rewrite the latter equation as

$$
f(B, T)=\frac{1}{\kappa} \int_{0}^{\kappa} f_{\frac{K \varepsilon(x)}{4}}(B, T) \mathrm{d} x,
$$

where $\kappa$ is defined in Eq. 6.4). Since the function $\varepsilon(x)$ is monotonically increasing in the interval $[0, \kappa]$, performing the change of variables $J=\frac{K \varepsilon(x)}{4}$ in the latter integral we immediately obtain

$$
f(B, T)=\int_{\min \left(0, J_{0}\right)}^{\max \left(0, J_{0}\right)} \rho(J) f_{J}(B, T) \mathrm{d} J,
$$

where

$$
J_{0}=\frac{K}{4} \varepsilon(\kappa)= \begin{cases}\frac{K}{16}, & \text { for the HS chain } \\ \frac{K}{4}, & \text { for the PF chain } \\ \frac{K}{4}(\gamma+1), & \text { for the FI chain, }\end{cases}
$$

and

$$
\rho(J)=\frac{4}{\underset{32}{\kappa|K| \varepsilon^{\prime}(x)}} .
$$


Note that $J_{0}$ has the sign of $K$, so that the integration range in Eq. 7.5$]$ is $\left[0, J_{0}\right]$ in the ferromagnetic case and $\left[J_{0}, 0\right]$ in the antiferromagnetic one. Furthermore, from Eq. (7.7) it immediately follows that

$$
\int_{\min \left(0, J_{0}\right)}^{\max \left(0, J_{0}\right)} \rho(J) \mathrm{d} J=\frac{1}{\kappa} \int_{0}^{\kappa} \mathrm{d} \kappa=1 .
$$

Hence, by Eq. (7.5), the free energy of each of the spin chains (1.6)-(1.7) is the average of the free energy of an ensemble of standard Ising models weighted by the function $\rho(J)$ in Eq. (7.7). This function, which depends on the chain under consideration through the dispersion relation $\varepsilon(x)$, is easily computed. Indeed, for the HS chain we have

$$
\varepsilon(x)=x(1-x)=\frac{1}{4}-\left(x-\frac{1}{2}\right)^{2} \equiv \frac{4 J}{K},
$$

so that

$$
\varepsilon^{\prime}(x)=1-2 x=\sqrt{1-\frac{16 J}{K}} .
$$

From Eq. (7.7) with $\kappa=1 / 2$ we easily obtain

$$
\rho(J)=\frac{2}{\sqrt{K\left(\frac{K}{16}-J\right)}}=\frac{1}{2 \sqrt{J_{0}\left(J_{0}-J\right)}} .
$$

Similarly, in the case of the PF chain $\varepsilon^{\prime}(x)=1$ and therefore

$$
\rho(J)=\frac{4}{|K|}=\frac{1}{\left|J_{0}\right|}
$$

is constant. Finally, in the case of the FI chain we have

$$
\varepsilon(x)=x(\gamma+x)=\left(x+\frac{\gamma}{2}\right)^{2}-\frac{\gamma^{2}}{4}=\frac{4 J}{K},
$$

and therefore

$$
\varepsilon^{\prime}(x)=2 x+\gamma=\sqrt{\gamma^{2}+\frac{16 J}{K}} .
$$

Hence the weight function $\rho(J)$ is given in this case by

$$
\rho(J)=\frac{4}{\sqrt{K\left(\gamma^{2} K+16 J\right)}}=\frac{\gamma+1}{\sqrt{J_{0}\left(\gamma^{2} J_{0}+4(\gamma+1) J\right)}} .
$$

Introducing the dimensionless variables $\left|J_{0}\right| \rho(J)$ and $j \equiv J / J_{0}$ (where $j \in[0,1]$ in the ferromagnetic case and $j \in[-1,0]$ in the antiferromagnetic one) we can rewrite the previous formulas as

$$
\left|J_{0}\right| \rho(J)= \begin{cases}\frac{1}{2}(1-j)^{-1 / 2}, & \text { for the HS chain } \\ 1, & \text { for the PF chain } \\ (\gamma+1)\left[\gamma^{2}+4(\gamma+1) j\right]^{-1 / 2}, & \text { for the FI chain } .\end{cases}
$$




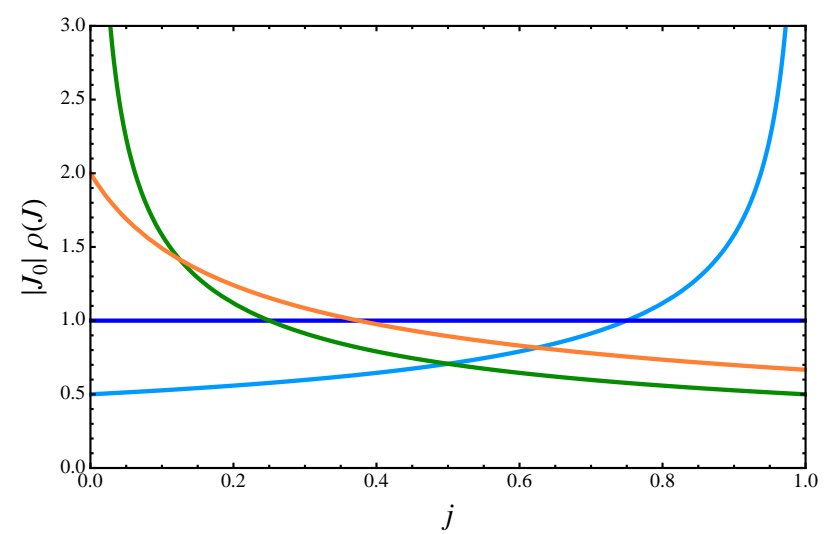

Figure 5: Normalized weight function $\left|J_{0}\right| \rho(J)$ versus dimensionless Ising coupling $j \equiv J / J_{0}$ for the HS (light blue), PF (blue) and FI ( $\gamma=0$, green; $\gamma=1$, orange) chains in the ferromagnetic case.

Equation (7.5) clearly implies that any thermodynamic quantity of a spin chain of HS type is the average of this quantity over an ensemble of standard Ising models with respect to the appropriate weight function $\rho(J)$ in Eqs. (7.8)-(7.10). In other words, a single spin chain of HS type is thermodynamically equivalent to a suitably weighted ensemble of standard Ising models. From this novel point of view, the fundamental difference between the three types of HS chains (1.6)(1.7) is the fact that the weight function $\rho(J)$ increases with the Ising coupling constant $J$ for the HS chain, is constant for the PF chain, and decreases with $J$ for the FI chain; cf. Fig. 5. The study of this weight function can also uncover some unexpected relations between the different families of chains of HS type. For instance, from Eq. (7.11) it follows that the normalized densities of the HS chain and the FI chain with $\gamma=0$ are dual, in the sense that they are related by the reflection $j \mapsto 1-j$ (cf. Fig. 5p.

\section{Conclusions and outlook}

In this paper we derive the thermodynamics of the three families of spin chains of HaldaneShastry type (1.6)-(1.7) in a unified way. The main idea behind our approach is to exploit the equivalence of these chains with a corresponding family of Ising-like inhomogeneous vertex models. In the absence of an external magnetic field, this fact had already been conjectured by Frahm [5] and by Frahm and Inozemtsev [6], and was recently established in a rigorous way in Ref. [35]. In this paper we generalize the results of the latter work to the case of an arbitrary magnetic field. Using the equivalence between spin chains of HS type and inhomogeneous vertex models, in the case of spin $1 / 2$ we are able to compute the large $N$ limit of the chains' canonical partition function by means of the transfer matrix method. We obtain in this way an explicit unified expression of the free energy per site, which we systematically use for a detailed analysis of the thermodynamic properties. In particular, we deduce asymptotic formulas for the relevant thermodynamic quantities in both the zero magnetic field and the zero temperature limits. Finally, we use the explicit formula for the free energy to show that spin chains of HS type are thermodynamically equivalent to a suitably weighted average of one-dimensional (homogeneous) Ising models. 
As remarked throughout the preceding sections, some of the formulas for the thermodynamic quantities of the HS-type chains derived in this paper have previously appeared in the literature, especially in the case of the original Haldane-Shastry chain. It should be stressed, however, that our approach to the thermodynamics of these chains differs from previous related work in two essential ways. First of all, we treat all three families of spin chains of HS type in a unified way via their dispersion relation $\varepsilon(x)$ (cf. (4.17)). As discussed in the previous section, this is possible due to the equivalence of these models (in the thermodynamic limit) to an inhomogeneous Ising model with couplings proportional to the values of the dispersion relation at the points $x_{i} \equiv i / N$, $1 \leqslant i \leqslant N-1$. On a more technical level, our method for studying the thermodynamics of spin chains of HS type is simply based on the evaluation of the canonical partition function in the thermodynamic limit through the standard transfer matrix approach. We thus bypass the more complex analysis relying on the equivalence of these models to an ideal gas of spinons used by Haldane to compute the thermodynamic functions of the original HS chain [23, 43], which requires the use of the grand canonical ensemble.

The results presented in this paper can be generalized in two different directions. On the one hand, it should be possible to apply our analysis to general vertex models of the form 4.2., in which the coupling $\varepsilon_{i}$ between consecutive spins is an arbitrary polynomial (or even a $C^{1}$ function) in the variable $x_{i}, 1 \leqslant i \leqslant N-1$. These models, which have been recently studied by Basu-Mallick and collaborators [35, 49], share certain basic properties with spin chains of HS-type; for instance, their energies are normally distributed in the thermodynamic limit. On the other hand, it should also be of interest to extend our results to other types of HS chains, particularly those associated with the $B C_{N}$ and $D_{N}$ root systems (see, e.g., [8-11]). The first step in this direction would be to ascertain whether these spin chains are isospectral to suitable vertex models analogous to 4.2 . Work on these and related topics, which is presently going on, shall be presented in subsequent publications.

\section{Acknowledgments}

This work was supported in part by the MICINN and the UCM-Banco Santander under grants no. FIS2011-22566 and GR35/10-A-910556.

\section{Appendix A. Error term in the asymptotic formulas 5.22)- 5.23}

Let us start with the error term in Eq. 5.22. We must show that

$$
\int_{0}^{R} \frac{t^{2} \mathrm{e}^{-t}}{\left(1+\mathrm{e}^{-t}\right)^{2}} \frac{\mathrm{d} t}{\sqrt{1-\frac{t}{R}}}-\int_{0}^{\infty} \frac{t^{2} \mathrm{e}^{-t}}{\left(1+\mathrm{e}^{-t}\right)^{2}} \mathrm{~d} t=\mathrm{O}\left(R^{-1}\right),
$$

where we have set $R=\lambda / 4$. Note first of all that the LHS in the previous equation can be written as

$$
\int_{0}^{R} \frac{t^{2} \mathrm{e}^{-t}}{\left(1+\mathrm{e}^{-t}\right)^{2}}\left[\left(1-\frac{t}{R}\right)^{-1 / 2}-1\right] \mathrm{d} t-\int_{R}^{\infty} \frac{t^{2} \mathrm{e}^{-t}}{\left(1+\mathrm{e}^{-t}\right)^{2}} \mathrm{~d} t,
$$

where the last term is easily seen to be $\mathrm{O}\left(R^{2} \mathrm{e}^{-R}\right)$. Hence we need only show that

$$
\int_{0}^{R} \frac{t^{2} \mathrm{e}^{-t}}{\left(1+\mathrm{e}^{-t}\right)^{2}}\left[\left(1-\frac{t}{R}\right)^{-1 / 2}-1\right] \mathrm{d} t=\mathrm{O}\left(R^{-1}\right) .
$$


Using the identity

$$
\left(1-\frac{t}{R}\right)^{-1 / 2}-1=2 R \frac{\mathrm{d}}{\mathrm{d} t}\left(1-\sqrt{1-\frac{t}{R}}-\frac{t}{2 R}\right)
$$

and integrating by parts we obtain

$$
\begin{aligned}
\int_{0}^{R} \frac{t^{2} \mathrm{e}^{-t}}{\left(1+\mathrm{e}^{-t}\right)^{2}} & {\left[\left(1-\frac{t}{R}\right)^{-1 / 2}-1\right] \mathrm{d} t } \\
& =2 R \int_{0}^{R}\left(1-\sqrt{1-\frac{t}{R}}-\frac{t}{2 R}\right) g(t) \mathrm{e}^{-t} \mathrm{~d} t+\mathrm{O}\left(R^{3} \mathrm{e}^{-R}\right)
\end{aligned}
$$

where

The elementary inequality

$$
g(t)=\frac{t^{2}-2 t}{\left(1+\mathrm{e}^{-t}\right)^{2}}-\frac{2 t^{2} \mathrm{e}^{-t}}{\left(1+\mathrm{e}^{-t}\right)^{3}}
$$

$$
1-\sqrt{1-s}-\frac{s}{2} \leqslant \frac{s^{2}}{2}, \quad 0 \leqslant s \leqslant 1,
$$

implies that

$$
0 \leqslant \int_{0}^{R} \frac{t^{2} \mathrm{e}^{-t}}{\left(1+\mathrm{e}^{-t}\right)^{2}}\left[\left(1-\frac{t}{R}\right)^{-1 / 2}-1\right] \mathrm{d} t \leqslant \frac{1}{R} \int_{0}^{R} t^{2} g(t) \mathrm{e}^{-t} \mathrm{~d} t+\mathrm{O}\left(R^{3} \mathrm{e}^{-R}\right)=\mathrm{O}\left(R^{-1}\right),
$$

since $g(t)=\mathrm{O}\left(t^{2}\right)$.

Consider next the error term in Eq. 5.23. We must now show that

$$
\int_{0}^{\infty} \frac{t^{2} \mathrm{e}^{-t}}{\left(1+\mathrm{e}^{-t}\right)^{2}} \mathrm{~d} t-\int_{0}^{(\gamma+1) \lambda} \frac{t^{2} \mathrm{e}^{-t}}{\left(1+\mathrm{e}^{-t}\right)^{2}} \frac{\mathrm{d} t}{\sqrt{1+\frac{4 t}{\gamma^{2} \lambda}}}=\mathrm{O}\left(\lambda^{-1}\right)
$$

or equivalently that

$$
\int_{0}^{(\gamma+1) \lambda} \frac{t^{2} \mathrm{e}^{-t}}{\left(1+\mathrm{e}^{-t}\right)^{2}}\left[1-\left(1+\frac{4 t}{\gamma^{2} \lambda}\right)^{-1 / 2}\right] \mathrm{d} t=\mathrm{O}\left(\lambda^{-1}\right)
$$

since

$$
\int_{(\gamma+1) \lambda}^{\infty} \frac{t^{2} \mathrm{e}^{-t}}{\left(1+\mathrm{e}^{-t}\right)^{2}} \mathrm{~d} t=\mathrm{O}\left(\lambda^{2} \mathrm{e}^{-(\gamma+1) \lambda}\right) .
$$

Our assertion follows immediately from the elementary inequality

$$
1-(1+s)^{-1 / 2} \leqslant \frac{s}{2}, \quad s>0,
$$

which implies that

$$
0 \leqslant \int_{0}^{(\gamma+1) \lambda} \frac{t^{2} \mathrm{e}^{-t}}{\left(1+\mathrm{e}^{-t}\right)^{2}}\left[1-\left(1+\frac{4 t}{\gamma^{2} \lambda}\right)^{-1 / 2}\right] \mathrm{d} t \leqslant \frac{2}{\gamma^{2} \lambda} \int_{0}^{(\gamma+1) \lambda} \frac{t^{3} \mathrm{e}^{-t}}{\left(1+\mathrm{e}^{-t}\right)^{2}}=\mathrm{O}\left(\lambda^{-1}\right)
$$

on account of the convergent character of the integral

$$
\int_{0}^{\infty} \frac{t^{3} \mathrm{e}^{-t}}{\left(1+\mathrm{e}^{-t}\right)^{2}} \mathrm{~d} t
$$




\section{Appendix B. Computation of the definite integrals 6.25}

Consider the definite integral

$$
I(x ; \alpha)=\int_{0}^{x} \log \left(\frac{1+\sqrt{1+4 \mathrm{e}^{\alpha t}}}{2}\right) \mathrm{d} t,
$$

with $\alpha^{2}=1$. Performing the change of variable

$$
z=\frac{1}{2}\left(1-\sqrt{1+4 \mathrm{e}^{\alpha t}}\right)
$$

and taking into account that

$$
\operatorname{Li}_{2}\left(\frac{1-\sqrt{5}}{2}\right)=\frac{1}{2} \log ^{2}\left(\frac{1+\sqrt{5}}{2}\right)-\frac{\pi^{2}}{15}
$$

(cf. Ref. [44]), we readily obtain

$$
\begin{aligned}
\alpha I(x ; \alpha) & =\int_{\frac{1}{2}(1-\sqrt{5})}^{\frac{1}{2}\left(1-\sqrt{1+4 \mathrm{e}^{\alpha x}}\right)}\left[\frac{\log (1-z)}{z}-\frac{\log (1-z)}{1-z}\right] \mathrm{d} z \\
& =\frac{1}{2} \log ^{2}\left(\frac{1+\sqrt{1+4 \mathrm{e}^{\alpha x}}}{2}\right)-\operatorname{Li}_{2}\left(\frac{1-\sqrt{1+4 \mathrm{e}^{\alpha x}}}{2}\right)-\frac{\pi^{2}}{15} .
\end{aligned}
$$

Hence

$$
I_{1}=I(\infty ;-1)=\frac{\pi^{2}}{15}
$$

As to the second integral in Eq. 6.25 , we first note that

$$
I_{2}=\lim _{x \rightarrow \infty}\left(I(x ; 1)-\frac{x^{2}}{4}\right) .
$$

From eq. B.1] and the dilogarithm identity [44]

$$
-\mathrm{Li}_{2}(1-z)=\mathrm{Li}_{2}\left(1-\frac{1}{z}\right)+\frac{1}{2} \log ^{2} z
$$

it follows that

$$
I(x ; 1)=\log ^{2}\left(\frac{1+\sqrt{1+4 \mathrm{e}^{x}}}{2}\right)+\operatorname{Li}_{2}\left(1-\frac{2}{1+\sqrt{1+4 \mathrm{e}^{x}}}\right)-\frac{\pi^{2}}{15},
$$

and therefore

$$
\begin{aligned}
I_{2} & =\mathrm{Li}_{2}(1)-\frac{\pi^{2}}{15}+\lim _{x \rightarrow \infty}\left[\log ^{2}\left(\frac{1+\sqrt{1+4 \mathrm{e}^{x}}}{2}\right)-\frac{x^{2}}{4}\right] \\
& =\frac{\pi^{2}}{10}+\lim _{x \rightarrow \infty}\left[\log ^{2}\left(\frac{1+\sqrt{1+4 \mathrm{e}^{x}}}{2}\right)-\frac{x^{2}}{4}\right],
\end{aligned}
$$


where we have made use of the equality $\operatorname{Li}_{2}(1)=\zeta(2)=\pi^{2} / 6$ (cf. Ref. [44]). Since

$$
\log \left(\frac{1+\sqrt{1+4 \mathrm{e}^{x}}}{2}\right)=\frac{x}{2}+\mathrm{O}\left(\mathrm{e}^{-x / 2}\right),
$$

the limit in Eq. (B.3) vanishes, and we finally obtain

$$
I_{2}=\frac{\pi^{2}}{10}
$$

\section{References}

[1] A. P. Polychronakos, J. Phys. A: Math. Gen. 39 (2006) 12793-12845.

[2] F. D. M. Haldane, Phys. Rev. Lett. 60 (1988) 635-638.

[3] B. S. Shastry, Phys. Rev. Lett. 60 (1988) 639-642.

[4] A. P. Polychronakos, Phys. Rev. Lett. 70 (1993) 2329-2331.

[5] H. Frahm, J. Phys. A: Math. Gen. 26 (1993) L473-L479.

[6] H. Frahm, V. I. Inozemtsev, J. Phys. A: Math. Gen. 27 (1994) L801-L807.

[7] T. Yamamoto, O. Tsuchiya, J. Phys. A: Math. Gen. 29 (1996) 3977-3984.

[8] A. Enciso, F. Finkel, A. González-López, M. A. Rodríguez, Nucl. Phys. B 707 (2005) 553-576.

[9] J. C. Barba, F. Finkel, A. González-López, M. A. Rodríguez, Phys. Rev. B 77 (2008) 214422(10),

[10] B. Basu-Mallick, F. Finkel, A. González-López, Nucl. Phys. B 812 (2009) 402-423.

[11] B. Basu-Mallick, F. Finkel, A. González-López, Nucl. Phys. B 843 (2011) 505-553.

[12] F. D. M. Haldane, Phys. Rev. Lett. 67 (1991) 937-940.

[13] F. Gebhard, A. E. Ruckenstein, Phys. Rev. Lett. 68 (1992) 244-247.

[14] M. Fowler, J. A. Minahan, Phys. Rev. Lett. 70 (1993) 2325-2328.

[15] D. Bernard, M. Gaudin, F. D. M. Haldane, V. Pasquier, J. Phys. A: Math. Gen. 26 (1993) 5219-5236.

[16] Z. N. C. Ha, F. D. M. Haldane, Phys. Rev. B 46 (1992) 9359-9368.

[17] J. A. Minahan, A. P. Polychronakos, Phys. Lett. B 302 (1993) 265-270.

[18] V. I. Inozemtsev, Phys. Scr. 53 (1996) 516-520.

[19] J. C. Barba, F. Finkel, A. González-López, M. A. Rodríguez, Europhys. Lett. 83 (2008) 27005(6).

[20] J. C. Barba, F. Finkel, A. González-López, M. A. Rodríguez, Phys. Rev. E 80 (2009) 047201(4).

[21] B. Basu-Mallick, H. Ujino, M. Wadati, J. Phys. Soc. Jpn. 68 (1999) 3219-3226.

[22] B. Basu-Mallick, N. Bondyopadhaya, Nucl. Phys. B 757 (2006) 280-302.

[23] F. D. M. Haldane, Phys. Rev. Lett. 66 (1991) 1529-1532.

[24] B. Basu-Mallick, N. Bondyopadhaya, D. Sen, Nucl. Phys. B 795 (2008) 596-622.

[25] J. I. Cirac, G. Sierra, Phys. Rev. B 81 (2010) 104431(4).

[26] N. Beisert, C. Kristjansen, M. Staudacher, Nucl. Phys. B 664 (2003) 131-184.

[27] T. Bargheer, N. Beisert, F. Loebbert, J. Phys. A: Math. Theor. 42 (2009) 285205(58).

[28] M. Greiter, Phys. Rev. B 79 (2009) 064409(5).

[29] K. Hikami, Nucl. Phys. B 441 (1995) 530-548.

[30] B. Basu-Mallick, Nucl. Phys. B 540 (1999) 679-704.

[31] N. Beisert, D. Erkal, J. Stat. Mech. 0803 (2008) P03001.

[32] A. P. Polychronakos, Nucl. Phys. B 419 (1994) 553-566.

[33] F. Finkel, A. González-López, Phys. Rev. B 72 (2005) 174411(6).

[34] J. C. Barba, F. Finkel, A. González-López, M. A. Rodríguez, Nucl. Phys. B 839 (2010) 499-525.

[35] B. Basu-Mallick, N. Bondyopadhaya, K. Hikami, SIGMA 6 (2010) 091-13.

[36] F. D. M. Haldane, Z. N. C. Ha, J. C. Talstra, D. Bernard, V. Pasquier, Phys. Rev. Lett. 69 (1992) 2021-2025.

[37] F. Calogero, J. Math. Phys. 12 (1971) 419-436.

[38] E. Corrigan, R. Sasaki, J. Phys. A: Math. Gen. 35 (2002) 7017-7061.

[39] A. Enciso, F. Finkel, A. González-López, M. A. Rodríguez, J. Nonlin. Math. Phys. 15 (2008) 155-165.

[40] A. Enciso, Spin models of Calogero-Sutherland type and associated spin chains, 2009. Ph.D. Thesis, Universidad Complutense de Madrid (arXiv:0906.1167 1 [math-ph]).

[41] F. Finkel, D. Gómez-Ullate, A. González-López, M. A. Rodríguez, R. Zhdanov, Commun. Math. Phys. 221 (2001) 477-497.

[42] I. G. Macdonald, Symmetric Functions and Hall Polynomials, Oxford University Press, Oxford, 1995. 
[43] D. Bernard, V. Pasquier, D. Serban, A one-dimensioal ideal gas of spinons, or some exact results on the XXX spin chain with long range interaction, 1993. ArXiv:hep-th/9311013v1.

[44] L. Lewin, Polylogarithms and associated functions, North Holland, New York, 1981

[45] M. Abramowitz, I. A. Stegun, Handbook of Mathematical Functions, Dover, New York, ninth edition, 1970.

[46] G. Mussardo, Statistical Field Theory: an Introduction to Exactly Solved Models in Statistical Physics, Oxford University Press, Oxford, 2010.

[47] A. Erdélyi, Asymptotic Expansions, Dover, New York, 1956.

[48] R. J. Baxter, Exactly Solved Models in Statistical Mechanics, Academic Press, London, 1982.

[49] P. Banerjee, B. Basu-Mallick, Level density distribution for one-dimensional vertex models related to HaldaneShastry like spin chains, 2011. ArXiv:1111.4376v2 [cond-mat.stat-mech]. 